Article

\title{
Design, Synthesis and Anti-HIV Integrase Evaluation of $\mathrm{N}$-(5- Chloro-8-Hydroxy-2-Styrylquinolin-7-yl)Benzenesulfonamide Derivatives
}

\author{
Zi-Guo Jiao, Hong-Qiu He, Cheng-Chu Zeng *, Jian-Jun Tan, Li-Ming Hu and Cun-Xin Wang * \\ College of Life Science \& Bioengineering, Beijing University of Technology, Beijing, China; \\ E-Mail: jiaoziguo@emails.bjut.edu.cn (Z.-G.J.)
}

* Authors to whom correspondence should be addressed; E-Mails: zengcc@bjut.edu.cn (C-C.Z.); cxwang@bjut.edu.cn (C-X.W.); Tel.: +86-10-67396211; Fax: +86-10-67392001.

Received: 21 October 2009; in revised form: 8 December 2009 / Accepted: 14 December 2009 / Published: 16 March 2010

Abstract: Styrylquinoline derivatives are demonstrated to be HIV-1 integrase inhibitors.
On the basis of our previous CoMFA analysis of a series of styrylquinoline derivatives, $N$ -
[(2-substituted-styryl)-5-chloro- 8 -hydroxyquinolin-7-yl]-benzenesulfonamide derivatives
were designed and synthesized, and their possible HIV IN inhibitory activity was evaluated.

Keywords: styrylquinoline derivatives; HIV-1 IN inhibitors; $N$-(styryl-8-hydroxyquinolin7-yl)-benzenesulfonamide derivatives

\section{Introduction}

There has been an increasing attention in the development of HIV integrase (IN) as a promising anti-HIV target, due to the fact that HIV IN is essential in the replication of HIV-1 and there are no similar enzymes involved in human cellular functions [1,2]. Therefore, extensive efforts have been made, resulting in a large number of HIV IN inhibitors [3,4], among which polyhydroxylated styrylquinolines have displayed an antiviral activity in a de novo infection assay of CEM4 cells, thereby opening an exciting structural platform for the design of new anti-HIV drugs [5-8]. The structure-activity-relationship of these compounds reveals that for in vitro activity a carboxyl group at C-7, a hydroxyl group at C-8 (salicylic acid structure) in the quinoline subunit and an ancillary phenyl 
ring are required. For example, FZ-41 in Figure 1 is one of the typical styrylquinoline-type HIV IN inhibitors [5-8].

Figure 1. The design of $N$-(2-styrylquinolin-7-yl)benzenesulfonamides as potential HIV IN inhibitors.

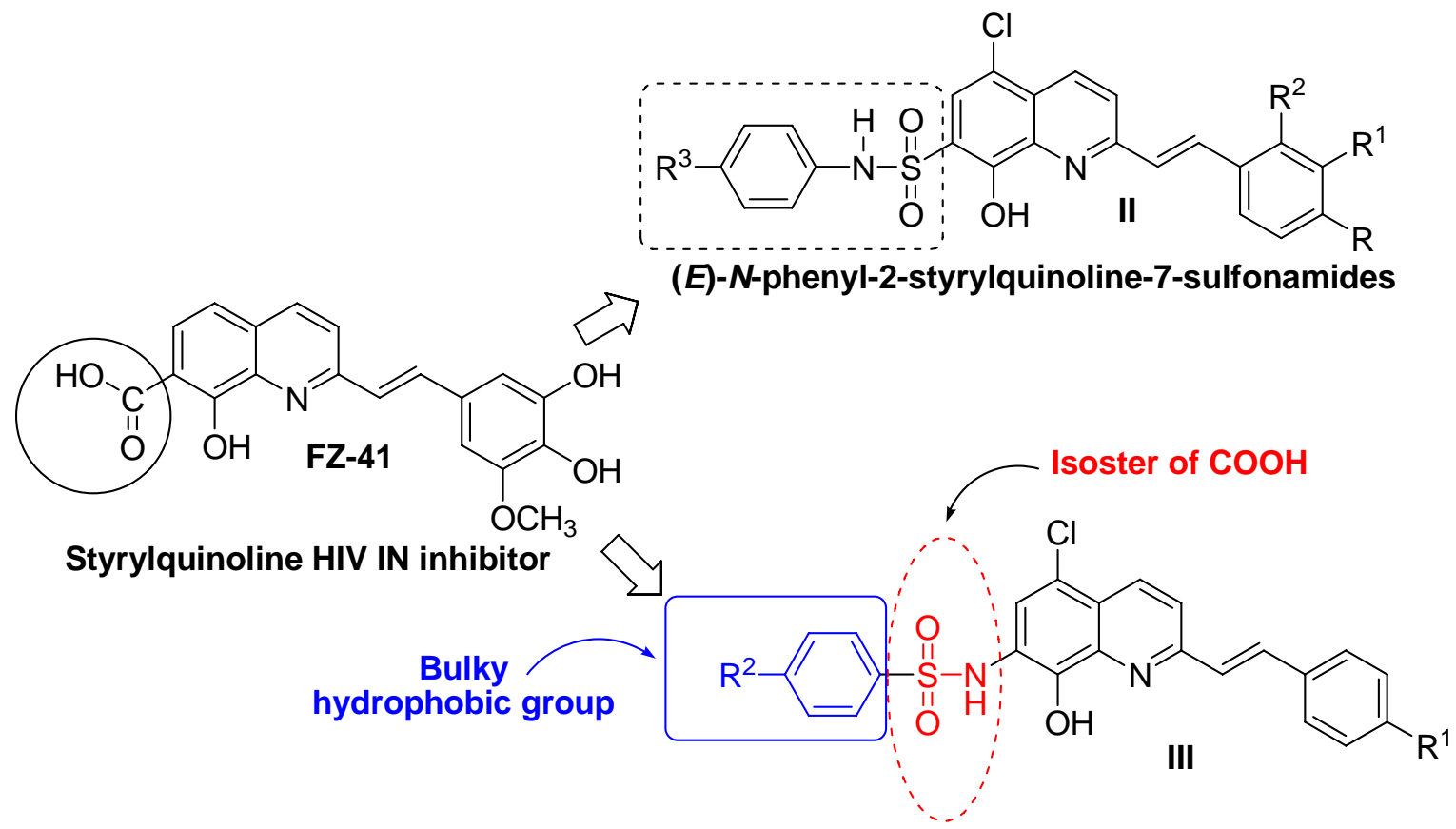

(E)-N-(2-styrylquinolin-7-yl)benzenesulfonamides

To better understand the pharmacophore properties of styrylquinoline derivatives and to further design potential HIV-IN inhibitors, we recently investigated 38 styrylquinoline derivatives employing a comparative molecular field analysis (CoMFA) method [9]. The results indicated that inhibitory activity should be increased if a bulky group was near the carboxyl group at C-7 in the quinoline ring. Simultaneously, the presence of H-bonding donor is favorable near the C-7 atom, which might form a stable H-bond with some protein residues.

On the basis of the above information, we decided to modify the basic scaffold of styrylquinolinetype HIV IN inhibitors by replacing the carboxylic functionality at the C-7 position with an aromatic sulfonamide as its bioisosteric functionality, bearing a bulky aromatic group and, meanwhile, maintaining the feature of the H-bonding donor. In addition, to increase the acidic properties of the C-8 phenolic $\mathrm{OH}$, a chloride atom was introduced at the $\mathrm{C}-5$ of the quinoline ring. Such a design resulted in the target $N$-phenyl-2-styrylquinoline-7-sulfonamides II and $N$-(styrylquinolin-7-yl)benzenesulfonamides III (Figure 1). Very recently, we have reported the synthesis and HIV IN screening of type II compounds [10]. In this work, we further describe the synthesis of type III compounds and their inhibitory activity against HIV IN. It should be pointed out that most of the work on the modification of styrylquinoline-type HIV IN concentrated on the modification of ancillary phenyl ring and linker unit. To the best of our knowledge, this is the first report concerning the replacement of salicyclic acid moiety of styrylquinoline HIV-IN inhibitors modified by sulfonamide. 


\section{Results and Discussion}

\section{Synthesis of N-(2-styrylquinolin-7-yl)benzenesulfonamide derivatives}

The synthesis of styrylquinoline-type HIV IN is well-documented in the literature [5-8,11]. The typical procedures are involved in the Perkin condensation of 2-methylquinoline and aromatic aldehyde [5-8], or the Wittig reaction between triphenylphosphonium salts and various benzaldehydes under basic conditions [11]. Obviously, the Perkin reaction process is more convenient than the Wittig reaction. Consequently, the synthesis of designed $N$-(5-chloro-8-hydroxy-2-styrylquinolin-7yl)benzenesulfonamide derivatives was carried out as shown in Scheme 1. The starting 5-chloroquinolin-8-ol (2) was easily prepared from 2-amino-4-chlorophenol (1) according to the known procedure [16]. The Perkin condensation between 2 and various aromatic aldehydes generated 5chloro-2-styrylquinolin-8-yl acetates $\mathbf{3}$ with pure $E$ geometry, which were hydrolyzed in pyridine/water to give 5-chloro-2-styrylquinolin-8-ols 4 . After nitration and reduction, compounds 4 was converted to 2-styryl-7-amino-5-chloroquinolin-8-ols 6 . The title styrylquinolin-7-ylbenzenesulfonamide derivatives III were finally produced in $16-56 \%$ yield after reaction with benzenesulfonyl chloride derivatives (Scheme 1 and Table 1).

Scheme 1. Synthesis of styrylquinolin-7-yl-benzenesulfonamide derivatives III.

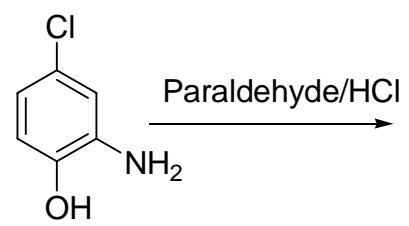

1

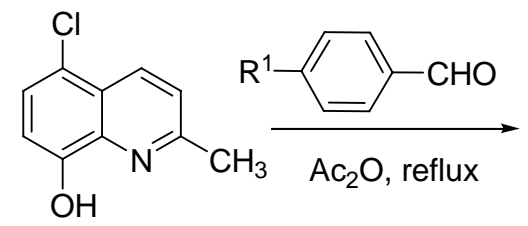

2

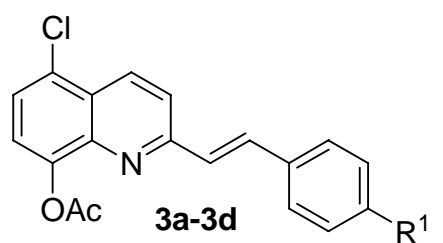

3a: $R^{1}=H ; 3 b: R^{1}=B r ;$

3c: $\mathrm{R}^{1}=\mathrm{OCH}_{3} ; \mathbf{3 d}: \mathrm{R}^{1}=$ benzyloxy<smiles>[R]c1ccc(/C=C/c2ccc3c(Cl)ccc(O)c3n2)cc1</smiles>

4a: $R^{1}=H ; 4 b: R^{1}=B r ;$ 4c: $\mathrm{R}^{1}=\mathrm{OCH}_{3} ; \mathbf{4 d}: \mathrm{R}^{1}=$ benzyloxy

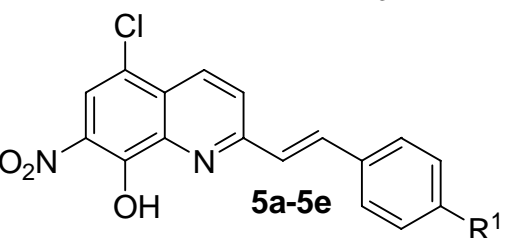

5a: $\mathrm{R}^{1}=\mathrm{H} ; \mathbf{5 b}: \mathrm{R}^{1}=\mathrm{Br} ; 5 \mathbf{c}: \mathrm{R}^{1}=\mathrm{OCH}_{3}$;

5d: $\mathrm{R}^{1}=$ benzyloxy; $5 e: \mathrm{R}^{1}=\mathrm{OH}$<smiles>[R]c1ccc(S(=O)(=O)Nc2cc(Cl)c3ccc(/C=C/c4cc(/C=C/c5ccc6c(Cl)cc(N)c(O)c6n5)ccc4[R])nc3c2O)cc1</smiles>

5a: $R^{1}=H ; 5 b: R^{1}=B r ;$

5c: $\mathrm{R}^{1}=\mathrm{OCH}_{3} ; 5 d: \mathrm{R}^{1}=\mathrm{OH}$

During the synthetic processes, the construction of the styryl scaffold by Perkin condensation reaction between 2-methylquinoline derivatives and various aldehydes was one of the key steps. According to a modified procedure [5,6], the 5-chloro-2-styrylquinolin-8-ols 4a-4d were isolated in $32-57 \%$ yield by column chromatography. It is noteworthy that the smooth Perkin condensation is 
likely dependent on the nature of the 2-methylquinoline component. In an initial attemption to construct the styryl scaffold, we tried to carry out Perkin conditions with 5-chloro-2-methyl-7-nitroquinolin-8-ol, but after refluxing in acetic anhydride for 7 days, no condensation product was detected and only 5-chloro-2-methyl-7-nitroquinolin-8-yl acetate was isolated (Scheme 2).

Scheme 2. Reaction between 5-chloro-2-methyl-7-nitroquinolin-8-ol and aldehydes.

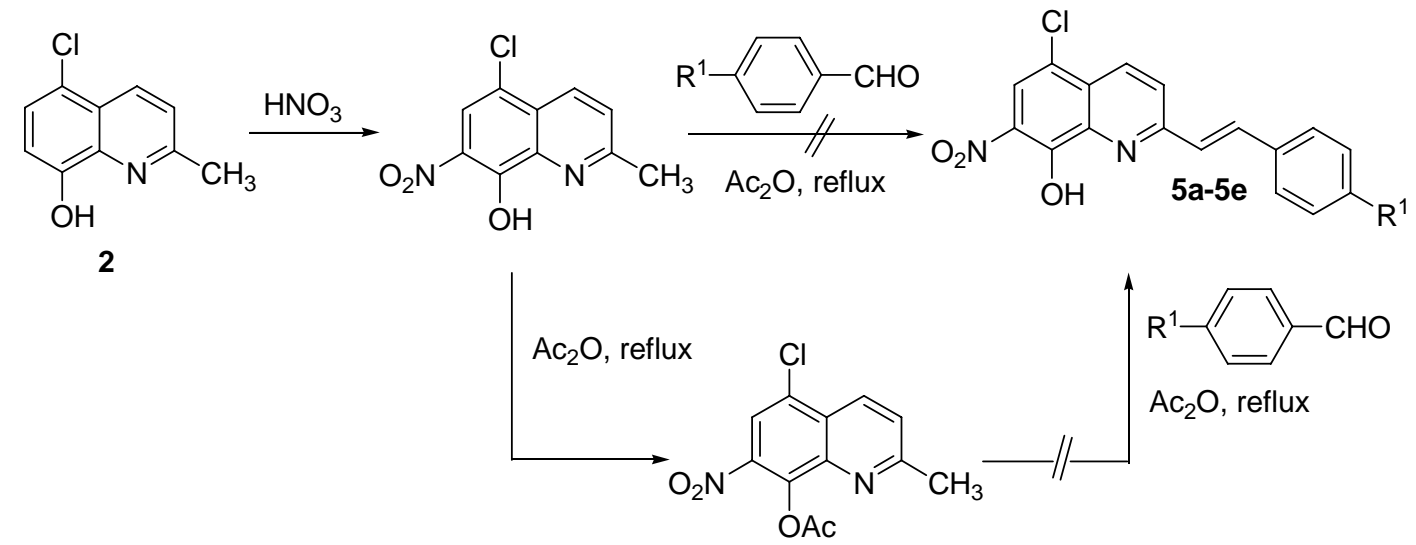

Selective sulfonylation reaction was also observed to be essential for the synthesis of title products III. In the presence of either pyridine or triethylamine, reaction of 2-styryl-7-amino-5-chloroquinolin8 -ols 6 and sulfonyl chloride always generated a mixture of $\mathrm{N}$ - and $\mathrm{O}$-sulfonylation products, that proved to be hard to separate by conventional techniques. These results are quite different from the case of $o$-aminophenol, which was reported to give selective $N$-tosylation or $O$-tosylation by using 1 equiv. of pyridine or triethylamine, respectively [12]. Finally, we found that utilizing DMAP as a catalyst and pyridine as the solvent, the desired sulfonamide analogues could be synthesized smoothly [13].

Table 1. The yields of styrylquinolin-7-yl-benzenesulfonamide derivatives III.

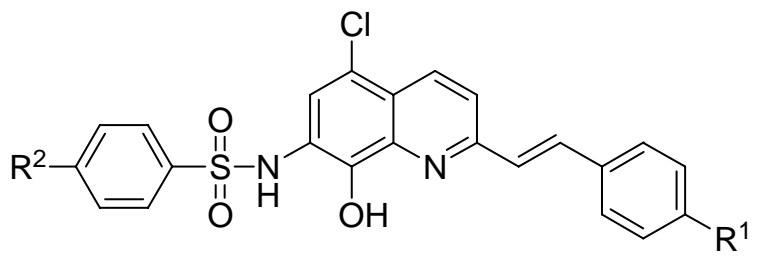

IIla-q

\begin{tabular}{cccccccc}
\hline No. & $\mathbf{R}^{\mathbf{1}}$ & $\mathbf{R}^{\mathbf{2}}$ & Yield & No. & $\mathbf{R}^{\mathbf{1}}$ & $\mathbf{R}^{\mathbf{2}}$ & Yield \\
\hline IIIa & $\mathrm{OCH}_{3}$ & $\mathrm{OCH}_{3}$ & $45 \%$ & IIIj & $\mathrm{Br}$ & $\mathrm{OCH}_{3}$ & $25 \%$ \\
IIIb & $\mathrm{OCH}_{3}$ & $\mathrm{CH}_{3}$ & $42 \%$ & IIIk & $\mathrm{Br}$ & $\mathrm{CH}_{3}$ & $51 \%$ \\
IIIc & $\mathrm{OCH}_{3}$ & $\mathrm{Cl}$ & $49 \%$ & IIIl & $\mathrm{Br}$ & $\mathrm{H}$ & $38 \%$ \\
IIId & $\mathrm{OCH}_{3}$ & $\mathrm{H}$ & $53 \%$ & IIIm & $\mathrm{Br}$ & $\mathrm{Cl}$ & $35 \%$ \\
IIIe & $\mathrm{H}$ & $\mathrm{Cl}$ & $52 \%$ & IIIn & $\mathrm{OH}$ & $\mathrm{OCH}_{3}$ & $33 \%$ \\
IIIf & $\mathrm{H}$ & $\mathrm{CH}_{3}$ & $51 \%$ & IIIo & $\mathrm{OH}$ & $\mathrm{CH}_{3}$ & $31 \%$ \\
IIIg & $\mathrm{H}$ & $\mathrm{OCH}_{3}$ & $54 \%$ & IIIp & $\mathrm{OH}$ & $\mathrm{H}$ & $25 \%$ \\
IIIh & $\mathrm{H}$ & $\mathrm{H}$ & $46 \%$ & IIIq & $\mathrm{OH}$ & $\mathrm{Cl}$ & $16 \%$ \\
IIII & $\mathrm{H}$ & $\mathrm{NO}_{2}$ & $56 \%$ & & & & \\
\hline
\end{tabular}


The structures of styrylquinolin-7-yl-benzenesulfonamide derivatives III were confirmed by ${ }^{1} \mathrm{H}-\mathrm{NMR},{ }^{13} \mathrm{C}-\mathrm{NMR}$, IR, and ESI-MS. Taking compound IIIa as an example, in its ${ }^{1} \mathrm{H}-\mathrm{NMR}$ spectrum, there are two AA'BB' systems coupling in the range of 7.02 and $8.23 \mathrm{ppm}$, attributed to the proton signals of two benzene rings. The ethenyl linker shows two signals at 7.29 and $8.23 \mathrm{ppm}$ (AB system) with a coupling constant of $16.0 \mathrm{~Hz}$. This observation indicates that the styrylquinoline scaffold is in a trans configuration. In addition, the signals of three protons in quinoline ring (C-3, C-4 and C-6) exhibited a couple of doublets and a singlet at slightly low field at 7.75 (doublet), 8.34 (doublet) and 7.56 (singlet) ppm, respectively. The structure of IIIa (as well as for all compounds) was also characterized by ESI-MS. Strong peaks at 496.8 and 518.8 were recorded, which correspond to $[\mathrm{M}+\mathrm{H}]^{+}$and $[\mathrm{M}+\mathrm{Na}]^{+}$.

\section{HIV IN inhibitory activity}

All title compounds IIIa-q were preliminarily tested against purified HIV IN to determine any inhibitory activity possessed on the strand transfer reaction of IN. Using the high-throughput format assay approach developed by us [14], the inhibition percentages of styrylquinolin-7-ylbenzenesulfonamide derivatives IIIa-q were calculated based on the positive (baicalein) and negative ( $10 \%$ DMSO) controls and are listed in Table 2. For comparison, the $\mathrm{IC}_{50}$ data of baicalein and FZ-41 were also included.

As shown in Table 2, compounds IIIn-IIIq, in which a free para-hydroxy group is present, showed higher inhibitory activity than that of the positive control, whereas, when the hydroxyl group was replaced by an electron-donating group (such as methoxy, compounds IIIa-IIId), hydrogen (IIIeIIIi), or an electron-withdrawing group (such as bromide, compounds IIIj-IIIm), only a moderate inhibitory rate was observed. This observation means that the free hydroxyl moiety of styrylquinolin7-yl-benzenesulfonamide derivatives is required for the inhibitory activity against HIV-IN. In addition, it was observed that the electron-withdrawing group at the para-position of benzenesulfonamide, such as nitro, may favor the inhibitory activity. For example, compound IIIi exhibits $96.7 \%$ inhibitory rate which decreases to $82.0 \%$ and $72.9 \%$ when the nitro was replaced by methyl (IIIf) and methoxy groups (IIIg), respectively. This results is not surprising because the electron-withdrawing group at the para-position of benzenesulfonamide moiety will increase the acidity of the benzenesulfonamide and lead to easier chelating with co-enzyme (mostly metallic ions), which is essential for the HIV IN inhibitory activity [15].

Table 2. Inhibitory rate of synthesized styrylquinolin-7-yl-benzenesulfonamide derivatives III.

\begin{tabular}{cccc}
\hline Compound & $\begin{array}{c}\text { Inhibitory rate \% } \\
\mathbf{( 0 . 0 5} \mathbf{~ m m o l} / \mathbf{L})\end{array}$ & Compound & $\begin{array}{c}\text { Inhibitory rate \% } \\
\mathbf{( 0 . 0 5} \mathbf{~ m m o l} / \mathbf{L})\end{array}$ \\
\hline Baicalein (positive & 100 & Negative control $(10 \%$ & 0 \\
control) & $\left(\mathrm{IC}_{50}=1.06 \mu \mathrm{M}\right)^{\mathrm{a}}$ & DMSO) & \\
FZ41 & $\left(\mathrm{IC}_{50}=0.7 \mu \mathrm{M}\right)^{\mathrm{b}}$ & IIIi & 96.7 \\
IIIa & 11.8 & IIIj & 53.6 \\
IIIb & 54.6 & IIIk & 59.5 \\
IIIc & 58.2 & IIIl & 74.7 \\
IIId & 60.3 & IIIm & 67.8 \\
\hline
\end{tabular}


Table 2. Cont.

\begin{tabular}{cccc}
\hline IIIe & 82.7 & IIIn & 101.0 \\
IIIf & 82.0 & IIIo & 100.6 \\
IIIg & 72.9 & IIIp & 101.4 \\
IIIh & 95.9 & IIIq & 101.4 \\
\hline
\end{tabular}

${ }^{\mathrm{a}}$ Value of $\mathrm{IC}_{50}$ cited from reference [14]; ${ }^{\mathrm{b}}$ Value of $\mathrm{IC}_{50}$ cited from reference [6].

\section{Experimental}

\section{General}

All solvents were of commercial quality and were dried and purified by conventional methods. Melting points (mp) were determined on an XT4A Electrothermal apparatus equipped with a microscope and are uncorrected. Infrared spectra (IR) were recorded as thin films on $\mathrm{KBr}$ plates with a Bruker IR spectrophotometer and are expressed in $v\left(\mathrm{~cm}^{-1}\right)$. The ${ }^{1} \mathrm{H}$ - and ${ }^{13} \mathrm{C}-\mathrm{NMR}$ spectra were obtained using an $\mathrm{AV} 400 \mathrm{M}$ Bruker spectrometer in $\mathrm{CDCl}_{3}$ or DMSO- $d_{6}$ with TMS as internal reference. The MS spectra (ESI) were recorded on a Bruker Esquire 6000 mass spectrometer.

\section{General procedure for the Synthesis of (E)-5-Chloro-2-styryl-substituted Quinolin-8-ol Derivatives 4}

A mixture of 5-chloro-2-methylquinolin-8-ol (2, $20 \mathrm{mmol})$ [16] and the appropriate benzaldehydes $(60 \mathrm{mmol})$ in acetic anhydride $(50 \mathrm{~mL})$ was heated under reflux for $48 \mathrm{hrs}$ and concentrated in vacuo. The black residue that formed was dissolved in a mixed solution of pyridine $(40 \mathrm{~mL})$ and water $(10 \mathrm{~mL})$, and the resulting solution was subjected to reflux for $3 \mathrm{hrs}$. After removal of pyridine, the formed solid was dissolved in $\mathrm{CH}_{2} \mathrm{Cl}_{2}$, washed three times by water, and dried over $\mathrm{MgSO}_{4}$. The solvent was removed under rotary evaporation. The desired compounds $\mathbf{4}$ were finally isolated by column chromatograph eluted using a mixture of petroleum ether and ethyl acetate.

(E)-5-Chloro-2-styrylquinolin-8-ol (4a): Yield: 50\%; mp: $146-147{ }^{\circ} \mathrm{C} ;{ }^{1} \mathrm{H}-\mathrm{NMR}\left(400 \mathrm{MHz}, \mathrm{CDCl}_{3}\right) \delta$ $7.08(\mathrm{~d}, 1 \mathrm{H}, J=8.0 \mathrm{~Hz}, \operatorname{Ar}-H), 7.33(\mathrm{~d}, 1 \mathrm{H}, J=16 \mathrm{~Hz},-\mathrm{CH}=\mathrm{CH}-), 7.36(\mathrm{t}, 1 \mathrm{H}, J=8.0 \mathrm{~Hz}, \operatorname{Ar}-H)$, 7.43 (t, 2H, $J=8.0 \mathrm{~Hz}, \operatorname{Ar}-H), 7.44$ (d, 1H, $J=8.0 \mathrm{~Hz}, \operatorname{Ar}-H), 7.64$ (d, 2H, $J=7.2 \mathrm{~Hz}, \operatorname{Ar}-H), 7.72$ (d, $1 \mathrm{H}, J=8.8 \mathrm{~Hz}$, pyridine- $H), 7.74(\mathrm{~d}, 1 \mathrm{H}, J=16.4 \mathrm{~Hz},-\mathrm{CH}=\mathrm{CH}-), 8.44(\mathrm{~d}, 1 \mathrm{H}, J=8.8 \mathrm{~Hz}$, pyridine$H) ;{ }^{13} \mathrm{C}-\mathrm{NMR}\left(100 \mathrm{MHz}, \mathrm{CDCl}_{3}\right) \delta 110.3,120.4,121.0,125.2,126.9,127.3,127.4,128.9,129.1$, 133.8, 135.4, 136.1, 151.1, 154.1; IR (KBr): v 3,382, 3,022, 1,628, 1,500, 1,451, 1,251, 1,198 cm ; ESI-MS: $m / z 281.8(\mathrm{M}+\mathrm{H})^{+}, 303.8(\mathrm{M}+\mathrm{Na})^{+}$.

(E)-2-(4-Bromostyryl)-5-chloroquinolin-8-ol (4b): Yield: 57\%; mp: 195-196 ${ }^{\circ} \mathrm{C} ;{ }^{1} \mathrm{H}-\mathrm{NMR}(400 \mathrm{MHz}$, $\left.\mathrm{CDCl}_{3}\right) \delta 7.08(\mathrm{~d}, 1 \mathrm{H}, J=8.0 \mathrm{~Hz}, \mathrm{Ar}-H), 7.30(\mathrm{~d}, 1 \mathrm{H}, J=16 \mathrm{~Hz},-\mathrm{CH}=\mathrm{CH}-), 7.44(\mathrm{~d}, 1 \mathrm{H}, J=8.4 \mathrm{~Hz}$, $\operatorname{Ar}-H), 7.48(\mathrm{~d}, 2 \mathrm{H}, J=8.4 \mathrm{~Hz}, \operatorname{Ar}-H), 7.54(\mathrm{~d}, 2 \mathrm{H}, J=8.8 \mathrm{~Hz}, \operatorname{Ar}-H), 7.65(\mathrm{~d}, 1 \mathrm{H}$, $J=16.4 \mathrm{~Hz},-\mathrm{C} H=\mathrm{CH}-), 7.68(\mathrm{~d}, 1 \mathrm{H}, J=8.8 \mathrm{~Hz}$, pyridine- $H), 8.23$ (s br, $1 \mathrm{H}, \mathrm{OH}), 8.44(\mathrm{~d}, 1 \mathrm{H}$, $J=8.8 \mathrm{~Hz}$, pyridine- $H$ ); IR (KBr): $v 3,434,1,638,1,586,1,557,1,497,1,458,1,392,1,312,1,172 \mathrm{~cm}^{-}$ ; ESI-MS: $m / z 359.6(\mathrm{M}+\mathrm{H})^{+}, 357.6\left(\mathrm{M}^{-}-\mathrm{H}\right)$. 
(E)-2-(4-Methoxystyryl)-5-chloroquinolin-8-ol (4c): Yield: $57 \% ; \quad \mathrm{mp}: \quad 195-196 \quad{ }^{\circ} \mathrm{C} ; \quad{ }^{1} \mathrm{H}-\mathrm{NMR}$ $\left(400 \mathrm{MHz}, \mathrm{CDCl}_{3}\right) \delta 3.86\left(\mathrm{~s}, 3 \mathrm{H}, \mathrm{OCH}_{3}\right), 6.94(\mathrm{dt}, 2 \mathrm{H}, J=8.8 \mathrm{~Hz}, J=2.0 \mathrm{~Hz}, \mathrm{Ar}-H), 7.06(\mathrm{~d}, 1 \mathrm{H}$, $J=8.0 \mathrm{~Hz}, \mathrm{Ar}-H), 7.20(\mathrm{~d}, 1 \mathrm{H}, J=16 \mathrm{~Hz},-\mathrm{CH}=\mathrm{C} H-), 7.41(\mathrm{~d}, 1 \mathrm{H}, J=8.4 \mathrm{~Hz}, \mathrm{Ar}-H), 7.57(\mathrm{dt}, 2 \mathrm{H}, J$ $=8.4 \mathrm{~Hz}, J=2.0 \mathrm{~Hz}$, Ar- $H), 7.68(\mathrm{~d}, 1 \mathrm{H}, J=8.8 \mathrm{~Hz}$, pyridine- $H), 7.68(\mathrm{~d}, 1 \mathrm{H}, J=17.6 \mathrm{~Hz},-\mathrm{CH}=\mathrm{C} H-$ ), $8.41(\mathrm{~d}, 1 \mathrm{H}, J=8.8 \mathrm{~Hz}$, pyridine- $H) ;{ }^{13} \mathrm{C}-\mathrm{NMR}\left(100 \mathrm{MHz}, \mathrm{CDCl}_{3}\right) \delta 55.4,110.4,114.4,120.4$, 121.0, 125.0, 125.3, 126.6, 128.8, 128.9, 133.5, 135.0, 138.4, 151.1, 154.6, 160.5; IR (KBr): v 3,411, 3,031, 2,934, 2,841, 1,603, 1,513, 1,460, 1,152 $\mathrm{cm}^{-1}$; ESI-MS: $m / z 312.0(\mathrm{M}+\mathrm{H})^{+}, 309.7\left(\mathrm{M}^{-}-\mathrm{H}\right)$.

(E)-2-(4-Benzyloxystyryl-5-chloroquinolin-8-ol (4d): Yield: $\quad 32 \%$;mp: $\quad 168-170 \quad{ }^{\circ} \mathrm{C} ; \quad{ }^{1} \mathrm{H}-\mathrm{NMR}$ $\left(400 \mathrm{MHz} \mathrm{CDCl}_{3}\right) \delta 5.12\left(\mathrm{~s}, 2 \mathrm{H},-\mathrm{CH}_{2}\right), 7.02(\mathrm{~d}, 2 \mathrm{H}, J=8.4 \mathrm{~Hz}, \mathrm{Ar}-H), 7.07(\mathrm{~d}, 1 \mathrm{H}, J=8.4 \mathrm{~Hz}, \mathrm{Ar}-$ $H$ ), $7.21(\mathrm{~d}, 1 \mathrm{H}, J=16.4 \mathrm{~Hz},-\mathrm{CH}=\mathrm{CH}-), 7.36$ (tt, $1 \mathrm{H}, J=7.2 \mathrm{~Hz}, J=2.4 \mathrm{~Hz}, \mathrm{Ar}-H)$, 7.39-7.47 (m, $5 \mathrm{H}, \operatorname{Ar}-H), 7.58(\mathrm{~d}, 2 \mathrm{H}, J=8.8 \mathrm{~Hz}, \operatorname{Ar}-H), 7.69(\mathrm{~d}, 1 \mathrm{H}, J=8.8 \mathrm{~Hz}$, pyridine- $H), 7.70(\mathrm{~d}, 1 \mathrm{H}, J=15.6$ $\mathrm{Hz},-\mathrm{CH}=\mathrm{C} H-), 8.45$ (d, $1 \mathrm{H}, J=8.8 \mathrm{~Hz}$, pyridine- $H$ ); IR (KBr): $v$ 3,370, 1,622, 1,601,1,508, 1,456, 1,307, 1,237, 1,169 $\mathrm{cm}^{-1}$; ESI-MS: $m / z 387.9(\mathrm{M}+\mathrm{H})^{+} 385.7\left(\mathrm{M}^{-}-\mathrm{H}\right)$.

General Procedure for the Synthesis of (E)-5-Chloro-7-nitro-2-styryl-substituted Quinolin-8-ol Derivatives 5a-d

To a three-necked flask $(250 \mathrm{~mL})$ charged with a mixed solution of nitric acid $(50 \mathrm{~mL}, 65-68 \%)$ and water $(50 \mathrm{~mL})$ and cooled by ice-water bath was added the appropriate 2-styryl substituted quinolin-8-ol 4a-d $(10 \mathrm{mmol})$. The reaction solution was stirred for $12 \mathrm{hrs}$ under ice-water cooling and then $24 \mathrm{hrs}$ at room temperature. After addition of water $(100 \mathrm{~mL})$, precipitate formed which was filtered, washed by water for three times and dried to generate the desired 7-nitro-2-styrylquinolin-8ols 5a-d.

(E)-5-Chloro-7-nitro-2-styrylquinolin-8-ol (5a): Yield: 90\%; mp: 170-172 ${ }^{\circ} \mathrm{C}$; ${ }^{1} \mathrm{H}-\mathrm{NMR}(400 \mathrm{MHz}$, DMSO-d $d_{6} \delta .40$ (t, $\left.1 \mathrm{H}, J=7.2 \mathrm{~Hz}, \operatorname{Ar}-H\right), 7.48$ (t, 2H, $\left.J=7.2 \mathrm{~Hz}, \operatorname{Ar}-H\right), 7.55(\mathrm{~d}, 1 \mathrm{H}, J=16 \mathrm{~Hz}$, $-\mathrm{CH}=\mathrm{C} H-), 7.73(\mathrm{~d}, 2 \mathrm{H}, J=7.6 \mathrm{~Hz}, \operatorname{Ar}-H), 8.09(\mathrm{~d}, 1 \mathrm{H}, J=8.8 \mathrm{~Hz}$, pyridine- $H), 8.13(\mathrm{~s}, 1 \mathrm{H}, \operatorname{Ar}-H)$, $8.35(\mathrm{~d}, 1 \mathrm{H}, J=16 \mathrm{~Hz},-\mathrm{C} H=\mathrm{C} H-), 8.53(\mathrm{~d}, 1 \mathrm{H}, J=8.8 \mathrm{~Hz}$, pyridine- $H), 11.66(\mathrm{~s}, \mathrm{br}, 1 \mathrm{H}, \mathrm{OH}) ;{ }^{13} \mathrm{C}-$ NMR $\left(100 \mathrm{MHz}, \mathrm{DMSO}-d_{6}\right) \delta 119.2,121.2,125.5,126.7,127.6,128.0,129.4,129.7,132.7,134.1$, 136.5, 137.7, 140.1, 150.0, 156.3; IR (KBr): $v$ 3,435, 1,568, 1,511, 1,328, 1,301, 1,252 cm ${ }^{-1}$; ESI-MS: $m / z 324.5\left(\mathrm{M}^{-}-\mathrm{H}\right)$.

(E)-2-(4-Bromostyryl)-5-chloro-7-nitroquinolin-8-ol (5b): Yield: 89\%; mp: $215-217{ }^{\circ} \mathrm{C} ;{ }^{1} \mathrm{H}-\mathrm{NMR}$ $\left(400 \mathrm{MHz}, \mathrm{DMSO}-d_{6}\right) \delta 7.58(\mathrm{~d}, 1 \mathrm{H}, J=16.4 \mathrm{~Hz},-\mathrm{CH}=\mathrm{CH}-), 7.67$ (s, 4H, Ar- $H$ ), 8.07 (d, 1H, $J=8.4$ $\mathrm{Hz}$, pyridine- $H), 8.13$ (s, $1 \mathrm{H}, \mathrm{Ar}-H), 8.32(\mathrm{~d}, 1 \mathrm{H}, J=16.4 \mathrm{~Hz},-\mathrm{C} H=\mathrm{C} H-), 8.54$ (d, $1 \mathrm{H}, J=8.4 \mathrm{~Hz}$, pyridine- $H$ ), $11.66(\mathrm{~s}, \mathrm{br}, 1 \mathrm{H}, \mathrm{OH}) ;{ }^{13} \mathrm{C}-\mathrm{NMR}\left(100 \mathrm{MHz}, \mathrm{DMSO}-d_{6}\right) \delta 119.3,121.3,122.8,125.6$, 127.5, 127.6, 129.8, 132.4, 132.7, 134.2, 135.8, 136.3, 140.2, 150.0, 156.0; IR (KBr): v 3,436, 1,631, 1,566, 1,518, 1,394, 1,344 $\mathrm{cm}^{-1}$; ESI-MS: $\mathrm{m} / z$ $404.5\left(\mathrm{M}^{-}-\mathrm{H}\right)$.

(E)-2-(4-Methoxystyryl)-5-chloro-7-nitroquinolin-8-ol (5c): Yield: 85\%; mp: $154-156{ }^{\circ} \mathrm{C}$; ${ }^{1} \mathrm{H}-\mathrm{NMR}$ $\left(400 \mathrm{MHz}, \mathrm{DMSO}-d_{6}\right) \delta 3.83\left(\mathrm{~s}, 3 \mathrm{H}, \mathrm{OCH}_{3}\right), 7.04$ (d, 2H, $\left.J=8.4 \mathrm{~Hz}, \mathrm{Ar}-H\right), 7.40$ (d, $1 \mathrm{H}, J=16 \mathrm{~Hz}$, 
- $\mathrm{C} H=\mathrm{C} H-), 7.68(\mathrm{~d}, 2 \mathrm{H}, J=8.0 \mathrm{~Hz}, \operatorname{Ar}-H), 8.05(\mathrm{~d}, 1 \mathrm{H}, J=8.4 \mathrm{~Hz}$, pyridine- $H), 8.11(\mathrm{~s}, 1 \mathrm{H}, \operatorname{Ar}-H)$, $8.29(\mathrm{~d}, 1 \mathrm{H}, J=16 \mathrm{~Hz},-\mathrm{C} H=\mathrm{C} H-), 8.50(\mathrm{~d}, 1 \mathrm{H}, J=8.4 \mathrm{~Hz}$, pyridine- $H), 11.48$ (s, br, $1 \mathrm{H}, \mathrm{OH})$; IR $(\mathrm{KBr}): v 3,436,2,968,1,595,1,567,1,514,1,380,1,256 \mathrm{~cm}^{-1}$; ESI-MS: $m / z 354.7\left(\mathrm{M}^{-}-\mathrm{H}\right)$.

(E)-2-(4-Benzyloxystyryl)-5-chloro-7-nitroquinolin-8-ol (5d): Yield: 94\%; mp: 207-208 ${ }^{\circ} \mathrm{C}$; ${ }^{1} \mathrm{H}-\mathrm{NMR}$ $\left(400 \mathrm{MHz}, \mathrm{DMSO}-d_{6}\right) \delta 5.18\left(\mathrm{~s}, 2 \mathrm{H},-\mathrm{CH}_{2^{-}}\right), 7.13(\mathrm{~d}, 2 \mathrm{H}, J=8.8 \mathrm{~Hz}, \mathrm{Ar}-H), 7.36(\mathrm{t}, 1 \mathrm{H}, J=8.8 \mathrm{~Hz}$, Ar- $H$ ), 7.42 (t, 2H, $J=7.2 \mathrm{~Hz}, \operatorname{Ar}-H), 7.42$ (d, 1H, $J=15.2 \mathrm{~Hz},-\mathrm{CH}=\mathrm{CH}-), 7.48$ (d, 2H, $J=6.8 \mathrm{~Hz}$, Ar- $H), 7.69(\mathrm{~d}, 2 \mathrm{H}, J=8.4 \mathrm{~Hz}, \operatorname{Ar}-H), 8.05(\mathrm{~d}, 1 \mathrm{H}, J=8.8 \mathrm{~Hz}$, pyridine- $H), 8.11(\mathrm{~s}, 1 \mathrm{H}, \operatorname{Ar}-H), 8.30$ $(\mathrm{d}, 1 \mathrm{H}, J=16 \mathrm{~Hz},-\mathrm{C} H=\mathrm{CH}-), 8.51(\mathrm{~d}, 1 \mathrm{H}, J=8.8 \mathrm{~Hz}$, pyridine- $H), 11.66(\mathrm{~s}, \mathrm{br}, 1 \mathrm{H}, \mathrm{OH})$; IR $(\mathrm{KBr}): v$ $3,433,1,566,1,513,1,385,1,330,1,237,1,176 \mathrm{~cm}^{-1}$; ESI-MS: $m / z 430.8\left(\mathrm{M}^{-}-\mathrm{H}\right)$.

(E)-2-(4-Hydroxystyryl)-5-chloro-7-nitroquinolin-8-ol (5e): To a 1:1 mixture of $\mathrm{HCl}$ and acetic acid $(30 \mathrm{~mL})$ in a round-bottle flask $(100 \mathrm{~mL})$ was added $\mathbf{5 d}(5 \mathrm{mmol})$. The mixture was heated under reflux for $3 \mathrm{hrs}$ till the completion of the reaction. After removal of solvent under reduced pressure, the yellow powder was washed with water to give compound 5e. ${ }^{1} \mathrm{H}-\mathrm{NMR}\left(400 \mathrm{MHz}, \mathrm{DMSO}-d_{6}\right) \delta 6.85$ $(\mathrm{d}, 2 \mathrm{H}, J=8.8 \mathrm{~Hz}, \mathrm{Ar}-H), 7.22(\mathrm{~d}, 1 \mathrm{H}, J=16 \mathrm{~Hz},-\mathrm{CH}=\mathrm{CH}-), 7.53(\mathrm{~d}, 2 \mathrm{H}, J=8.4 \mathrm{~Hz}, \mathrm{Ar}-H), 7.70$ (d, $1 \mathrm{H}, J=16.4 \mathrm{~Hz},-\mathrm{C} H=\mathrm{C} H-), 7.82(\mathrm{~d}, 1 \mathrm{H}, J=8.4 \mathrm{~Hz}$, pyridine- $H), 7.96(\mathrm{~s}, 1 \mathrm{H}, \mathrm{Ar}-H), 8.13(\mathrm{~d}, 1 \mathrm{H}$, $J=8.4 \mathrm{~Hz}$, pyridine- $H$ ), $9.81(\mathrm{~s}, \mathrm{br}, 1 \mathrm{H}, \mathrm{O} H) ;{ }^{13} \mathrm{C}-\mathrm{NMR}\left(100 \mathrm{MHz}, \mathrm{DMSO}-d_{6}\right) \delta 109.0,116.3,122.8$, 123.7, 125.3, 127.8, 128.6, 129.1, 131.1, 132.8, 134.2, 148.4, 153.8, 158.8, 164.2; IR (KBr): v 3,436, 2,979, 2,681, 1,635, 1,604, 1,555, 1,515, 1,466, 1,278, 1,247, 1,170 cm ${ }^{-1}$; ESI-MS: $m / z 340.7$ (M-H).

General Procedure for the Synthesis of (E)-7-Amino-5-chloro-2-styryl-substituted Quinolin-8-ol Derivatives 6a-d

To a suspension of (E)-5-chloro-7-nitro-2-styryl substituted quinolin-8-ol 5a-d (5 mmol) in methanol $(50 \mathrm{~mL})$ and water $(50 \mathrm{~mL})$ was added 40 equivalents of sodium dithionite. The reaction mixture was stirred for $24 \mathrm{hrs}$ at room temperature and then quenched by adding water $(100 \mathrm{~mL})$. The formed precipitate was filtered, washed and dried to get desired (E)-7-amino-5-chloro-2-styryl substituted quinolin-8-ol derivatives 6a-d.

(E)-7-Amino-5-chloro-2-styrylquinolin-8-ol (6a): Yield: 86\%; mp: 193-195 ${ }^{\circ} \mathrm{C}$; ${ }^{1} \mathrm{H}-\mathrm{NMR}(400 \mathrm{MHz}$, DMSO- $\left.d_{6}\right) \delta 5.38\left(\mathrm{~s}, \mathrm{br}, 2 \mathrm{H}, \mathrm{N} H_{2}\right), 7.23(\mathrm{~s}, 1 \mathrm{H}, \mathrm{Ar}-H), 7.36(\mathrm{t}, 1 \mathrm{H}, J=7.2 \mathrm{~Hz}, \mathrm{Ar}-H), 7.44(\mathrm{~d}, 1 \mathrm{H}$, $J=16 \mathrm{~Hz},-\mathrm{C} H=\mathrm{CH}-), 7.46(\mathrm{t}, 2 \mathrm{H}, J=7.2 \mathrm{~Hz}, \mathrm{Ar}-H), 7.54(\mathrm{~d}, 1 \mathrm{H}, J=8.8 \mathrm{~Hz}$, pPyridine- $H), 7.72(\mathrm{~d}$, $2 \mathrm{H}, J=7.2 \mathrm{~Hz}, \mathrm{Ar}-H), 8.15(\mathrm{~d}, 1 \mathrm{H}, J=16.4 \mathrm{~Hz},-\mathrm{C} H=\mathrm{C} H-), 8.22(\mathrm{~d}, 1 \mathrm{H}, J=8.8 \mathrm{~Hz}$, pyridine- $H$ ), 9.03 (s, br, 1H, OH); IR (KBr): $v$ 3,436, 1,634, 1,508, 1,465, 1,290, 1,193 cm ${ }^{-1}$; ESI-MS: $m / z 286.9$ $(\mathrm{M}+\mathrm{H})^{+}, 294.5\left(\mathrm{M}^{-}-\mathrm{H}\right)^{-}$.

(E)-2-(4-Bromostyryl)-7-amino-5-chloroquinolin-8-ol (6b): Yield: 94\%;mp: 201-202 ${ }^{\circ} \mathrm{C}$; ${ }^{1} \mathrm{H}-\mathrm{NMR}$ $\left(400 \mathrm{MHz}, \mathrm{DMSO}-d_{6}\right) \delta 7.59$ (d, $\left.1 \mathrm{H}, J=16 \mathrm{~Hz},-\mathrm{CH}=\mathrm{CH}-\right), 7.66(\mathrm{~s}, 1 \mathrm{H}, \mathrm{OH}), 7.68(\mathrm{~s}, 4 \mathrm{H}, \mathrm{Ar}-H), 8.08$ $(\mathrm{d}, 1 \mathrm{H}, J=8.8 \mathrm{~Hz}$, pyridine- $H), 8.15(\mathrm{~s}, 1 \mathrm{H}, \mathrm{Ar}-H), 8.34(\mathrm{~d}, 1 \mathrm{H}, J=16.4 \mathrm{~Hz},-\mathrm{CH}=\mathrm{C} H-), 8.56(\mathrm{~d}, 1 \mathrm{H}$, $J=8.8 \mathrm{~Hz}$, pyridine- $H$ ); IR $(\mathrm{KBr}): v 3,432,3,080,1,628,1,566,1,516,1,342,1,180 \mathrm{~cm}^{-1}$; ESI-MS: $\mathrm{m} / \mathrm{z} 376.8(\mathrm{M}+\mathrm{H})^{+}$. 
(E)-2-(4-Methoxystyryl)-7-amino-5-chloroquinolin-8-ol (6c): Yield: 60\%; mp: $154-156{ }^{\circ} \mathrm{C} ;{ }^{1} \mathrm{H}-\mathrm{NMR}$ $\left(400 \mathrm{MHz}, \mathrm{DMSO}-d_{6}\right) \delta 3.81\left(\mathrm{~s}, 3 \mathrm{H}, \mathrm{OCH}_{3}\right), 5.42$ (s br, 2H, $\left.\mathrm{NH}_{2}\right), 7.02(\mathrm{~d}, 2 \mathrm{H}, J=8.4 \mathrm{~Hz}, \mathrm{Ar}-H)$, $7.21(\mathrm{~s}, 1 \mathrm{H}, \mathrm{Ar}-H), 7.29(\mathrm{~d}, 1 \mathrm{H}, J=16.4 \mathrm{~Hz},-\mathrm{CH}=\mathrm{CH}-), 7.49(\mathrm{~d}, 1 \mathrm{H}, J=8.4 \mathrm{~Hz}$, pyridine- $H), 7.66(\mathrm{~d}$, $2 \mathrm{H}, J=8.4 \mathrm{~Hz}, \mathrm{Ar}-H), 8.08$ (d, $1 \mathrm{H}, J=16 \mathrm{~Hz},-\mathrm{CH}=\mathrm{CH}-), 8.19$ (d, $1 \mathrm{H}, J=8.8 \mathrm{~Hz}$, pyridine- $H$ ), 8.99 (s, br, 1H, OH); IR (KBr): $v$ 3,450, 3,376, 3,338, 1,606, 1,513, 1,305, 1,176 cm ${ }^{-1}$; ESI-MS: $m / z 326.9$ $(\mathrm{M}+\mathrm{H})^{+} 324.8\left(\mathrm{M}^{-}-\mathrm{H}\right)$.

(E)-2-(4-Hydroxystyryl)-7-amino-5-chloroquinolin-8-ol (6d): Yield: $30 \%$; mp: $>300{ }^{\circ} \mathrm{C}$; ${ }^{1} \mathrm{H}-\mathrm{NMR}$ $\left(400 \mathrm{MHz}, \mathrm{DMSO}-d_{6}\right) \delta 5.75$ (s, br, 2H, NH$\left.H_{2}\right), 6.85$ (d, 2H, $\left.J=8.4 \mathrm{~Hz}, \mathrm{Ar}-H\right), 7.22(\mathrm{~s}, 1 \mathrm{H}, \mathrm{Ar}-H), 7.30$ (d, $1 \mathrm{H}, J=16 \mathrm{~Hz},-\mathrm{CH}=\mathrm{CH}-), 7.54(\mathrm{~d}, 1 \mathrm{H}, J=8.4 \mathrm{~Hz}$, pyridine- $H), 7.55(\mathrm{~d}, 2 \mathrm{H}, J=8.4 \mathrm{~Hz}, \operatorname{Ar}-H)$, $8.03(\mathrm{~d}, 1 \mathrm{H}, J=16 \mathrm{~Hz},-\mathrm{CH}=\mathrm{C} H-), 8.23(\mathrm{~d}, 1 \mathrm{H}, J=8.4 \mathrm{~Hz}$, pyridine- $H$ ), 9.11 (s, br, $1 \mathrm{H}, \mathrm{OH}), 9.88$ (s, $1 \mathrm{H}, \mathrm{OH})$; IR (KBr): $v$ 3,413, 3,369, 1,621, 1,514, 1,279, 1,171 $\mathrm{cm}^{-1}$; ESI-MS: $m / z 312.9(\mathrm{M}+\mathrm{H})^{+}$, $310.7\left(\mathrm{M}^{-}-\mathrm{H}\right)^{-}$.

General Procedure for the Synthesis of (E)-N-(2-substituted-styryl)-5-chloro-8-hydroxyquinolin-7-yl)benzenesulfonamides III

To a flask $(50 \mathrm{~mL})$ charged with the appropriate compound $6(0.5 \mathrm{mmol})$ and DMAP $(0.05 \mathrm{mmol})$ dissolved in pyridine $(10 \mathrm{~mL})$ was added dropwise benzenesulfonic chloride $(0.55 \mathrm{mmol})$ in pyridine $(5 \mathrm{~mL})$. After addition, the reaction mixture was stirred for $2 \mathrm{hrs}$ at room temperature and then the solvent was removed under reduced pressure. The residue was dissolved in $\mathrm{CH}_{2} \mathrm{Cl}_{2}(50 \mathrm{~mL})$ and washed with water, dried by $\mathrm{MgSO}_{4}$. The desired product III was obtained after column chromatograph and then recrystallization using petroleum ether and ethyl acetate.

(E)-N-(2-(4-Methoxystyryl)-5-chloro-8-hydroxyquinolin-7-yl)-4-methoxybenzenesulfonamide (IIIa): Yield: 44\%; mp: $192-193{ }^{\circ} \mathrm{C}$; ${ }^{1} \mathrm{H}-\mathrm{NMR}\left(400 \mathrm{MHz}, \mathrm{DMSO}-d_{6}\right) \delta 3.77$ (s, 3H, OCH $), 3.81(\mathrm{~s}, 3 \mathrm{H}$, $\left.\mathrm{OCH}_{3}\right), 7.02(\mathrm{~d}, 2 \mathrm{H}, J=8.8 \mathrm{~Hz}, \operatorname{Ar}-H), 7.02(\mathrm{~d}, 2 \mathrm{H}, J=8.8 \mathrm{~Hz}, \operatorname{Ar}-H), 7.29$ (d, $1 \mathrm{H}, J=16 \mathrm{~Hz}$, $\mathrm{C} H=\mathrm{C} H-), 7.56(\mathrm{~s}, 1 \mathrm{H}, \mathrm{Ar}-H), 7.64(\mathrm{~d}, 2 \mathrm{H}, J=8.8 \mathrm{~Hz}, \mathrm{Ar}-H), 7.72(\mathrm{~d}, 2 \mathrm{H}, J=8.8 \mathrm{~Hz}, \mathrm{Ar}-H), 7.75(\mathrm{~d}$, $1 \mathrm{H}, J=8.8 \mathrm{~Hz}$, pyridine- $H), 8.23(\mathrm{~d}, 1 \mathrm{H}, J=16 \mathrm{~Hz},-\mathrm{C} H=\mathrm{C} H-), 8.34(\mathrm{~d}, 1 \mathrm{H}, J=8.8 \mathrm{~Hz}$, pyridine- $H)$, $9.91(\mathrm{~s}, \mathrm{br}, 2 \mathrm{H}, \mathrm{OH}, \mathrm{N} H) ;{ }^{13} \mathrm{C}-\mathrm{NMR}\left(100 \mathrm{MHz}, \mathrm{DMSO}-d_{6}\right) \delta 55.7,56.0,114.7,114.9,118.6,121.2$, 122.8, 124.3, 124.9, 129.3, 129.3, 129.3, 129.4, 132.6, 133.4, 136.3, 138.9, 145.4, 155.4, 160.5, 162.8; IR (KBr): $v$ 3,420, 3,293, 2,930, 1,620, 1,593, 1,512, 1,258, 1,158 cm $\mathrm{cm}^{-1}$ ESI-MS: $m / z 494.7\left(\mathrm{M}^{-}-1\right)$, $496.8\left(\mathrm{M}^{+}+1\right), 518.8\left(\mathrm{M}^{+}+\mathrm{Na}\right)$.

(E)-N-(2-(4-Methoxystyryl)-5-chloro-8-hydroxyquinolin-7-yl)-4-methylbenzenesulfonamide

(IIIb): Yield: 42\%; mp: 202-204 ${ }^{\circ} \mathrm{C}$; ${ }^{1} \mathrm{H}-\mathrm{NMR}\left(400 \mathrm{MHz}, \mathrm{DMSO}-d_{6}\right) \delta 2.32\left(\mathrm{~s}, 3 \mathrm{H}, \mathrm{CH}_{3}\right), 3.81(\mathrm{~s}, 3 \mathrm{H}$, $\left.\mathrm{OCH}_{3}\right), 7.02(\mathrm{~d}, 2 \mathrm{H}, J=8.8 \mathrm{~Hz}, \mathrm{Ar}-H), 7.29(\mathrm{~d}, 1 \mathrm{H}, J=16 \mathrm{~Hz},-\mathrm{CH}=\mathrm{CH}-), 7.31(\mathrm{~d}, 2 \mathrm{H}, J=8.0 \mathrm{~Hz}$, Ar-H), 7.57 (s, $1 \mathrm{H}, \operatorname{Ar}-H), 7.64$ (d, 2H, $J=8.4 \mathrm{~Hz}, \operatorname{Ar}-H), 7.69$ (d, 2H, $J=8.0 \mathrm{~Hz}, \operatorname{Ar}-H), 7.75(\mathrm{~d}, 1 \mathrm{H}$, $J=8.8 \mathrm{~Hz}$, pyridine- $H$ ), 8.24 (d, $1 \mathrm{H}, J=16 \mathrm{~Hz},-\mathrm{C} H=\mathrm{C} H-), 8.34(\mathrm{~d}, 1 \mathrm{H}, J=8.4 \mathrm{~Hz}$, pyridine- $H), 9.90$ (s, br, 1H, OH), 10.01 (s, br, $1 \mathrm{H}, \mathrm{N} H)$; ${ }^{13} \mathrm{C}-\mathrm{NMR}\left(100 \mathrm{MHz}, \mathrm{DMSO}-d_{6}\right) \delta 21.4,55.7,114.9,118.6$, $121.1,122.4,122.8,124.3,124.9,127.1,129.3,129.4,130.0,133.3,136.3,138.2,138.9,143.5,145.4$, 
155.4, 160.5; IR (KBr): $v$ 3,448, 3,290, 1,634, 1,589, 1,513, 1,461, 1,249, 1,158 cm $\mathrm{cm}^{-1}$ ESI-MS: $\mathrm{m} / \mathrm{z}$ $478.8\left(\mathrm{M}^{-}-1\right), 480.8\left(\mathrm{M}^{+}+1\right), 502.8\left(\mathrm{M}^{+}+\mathrm{Na}\right)$.

(E)-N-(2-(4-Methoxystyryl)-5-chloro-8-hydroxyquinolin-7-yl)-4-chlorobenzenesulfonamide

(IIIc):

Yield: 49\%; mp: $219-221{ }^{\circ} \mathrm{C}$; ${ }^{1} \mathrm{H}-\mathrm{NMR}\left(400 \mathrm{MHz}, \mathrm{DMSO}-d_{6}\right) \delta 3.81\left(\mathrm{~s}, 3 \mathrm{H}, \mathrm{OCH}_{3}\right), 7.02(\mathrm{~d}, 2 \mathrm{H}$, $J=8.8 \mathrm{~Hz}, \operatorname{Ar}-H), 7.30$ (d, $1 \mathrm{H}, J=16.4 \mathrm{~Hz},-\mathrm{CH}=\mathrm{CH}-), 7.54$ (s, $1 \mathrm{H}, \operatorname{Ar}-H), 7.60$ (d, 2H, $J=8.8 \mathrm{~Hz}$, $\operatorname{Ar}-H), 7.64(\mathrm{~d}, 2 \mathrm{H}, J=8.8 \mathrm{~Hz}, \operatorname{Ar}-H), 7.78(\mathrm{~d}, 2 \mathrm{H}, J=8.8 \mathrm{~Hz}, \operatorname{Ar}-H), 7.78(\mathrm{~d}, 1 \mathrm{H}, J=8.8 \mathrm{~Hz}$, pyridine- $H$ ), $8.23(\mathrm{~d}, 1 \mathrm{H}, J=16 \mathrm{~Hz},-\mathrm{C} H=\mathrm{CH}-), 8.36(\mathrm{~d}, 1 \mathrm{H}, J=8.8 \mathrm{~Hz}$, pyridine- $H), 9.90$ (s br, $1 \mathrm{H}$, $\mathrm{O} H$ ), 10.23 (s br, $1 \mathrm{H}, \mathrm{N} H$ ); ${ }^{13} \mathrm{C}-\mathrm{NMR}\left(100 \mathrm{MHz}, \mathrm{DMSO}-d_{6}\right) \delta 55.7,114.9,118.7,120.4,122.5,123.2$, 124.9, 125.2, 129.0, 129.3, 129.4, 129.7, 133.4, 136.4, 138.1, 139.0, 140.0, 146.2, 155.5, 160.5; IR $(\mathrm{KBr}): v$ 3,291, 1,632, 1,589, 1,512, 1,464, 1,327, 1,259, 1,161 cm $\mathrm{cm}^{-1}$ ESI-MS: $\mathrm{m} / z 499.7\left(\mathrm{M}^{-}-1\right)$, $500.8\left(\mathrm{M}^{+}+1\right)$.

(E)-N-(2-(4-Methoxystyryl)-5-chloro-8-hydroxyquinolin-7-yl)benzenesulfonamide (IIId): Yield: 53\%; mp: $237-238{ }^{\circ} \mathrm{C}$; ${ }^{1} \mathrm{H}-\mathrm{NMR}\left(400 \mathrm{MHz}, \mathrm{DMSO}-d_{6}\right) \delta 3.80\left(\mathrm{~s}, 3 \mathrm{H}, \mathrm{OCH}_{3}\right), 7.00(\mathrm{~d}, 2 \mathrm{H}, J=8.8 \mathrm{~Hz}, \mathrm{Ar}-$ $H), 7.27(\mathrm{~d}, 1 \mathrm{H}, J=16 \mathrm{~Hz},-\mathrm{CH}=\mathrm{C} H-), 7.51(\mathrm{t}, 2 \mathrm{H}, J=7.2 \mathrm{~Hz}, \mathrm{Ar}-H), 7.55(\mathrm{~s}, 1 \mathrm{H}, \mathrm{Ar}-H), 7.57(\mathrm{t}, 1 \mathrm{H}$, $J=7.2 \mathrm{~Hz}, \operatorname{Ar}-H), 7.62(\mathrm{~d}, 2 \mathrm{H}, J=8.8 \mathrm{~Hz}, \operatorname{Ar}-H), 7.73(\mathrm{~d}, 1 \mathrm{H}, J=8.8 \mathrm{~Hz}$, pyridine- $H), 7.81(\mathrm{~d}, 2 \mathrm{H}$, $J=8.8 \mathrm{~Hz}, \mathrm{Ar}-H), 8.22(\mathrm{~d}, 1 \mathrm{H}, J=16 \mathrm{~Hz},-\mathrm{C} H=\mathrm{C} H-), 8.31$ (d, $1 \mathrm{H}, J=8.8 \mathrm{~Hz}$, pyridine- $H$ ), 10.10 (s, br, $2 \mathrm{H}, \mathrm{OH}, \mathrm{N} H) ;{ }^{13} \mathrm{C}-\mathrm{NMR}\left(100 \mathrm{MHz}, \mathrm{DMSO}-d_{6}\right) \delta 55.7,114.8,118.7,120.9,122.3,122.9,124.5$, $124.9,127.0,129.3,129.4,129.5,133.2,133.3,136.3,138.9,141.0,145.7,155.5,160.5$; IR (KBr): $v$ 3,371, 3,241, 1,627, 1,513, 1,455, 1,329, 1,249, 1,172 $\mathrm{cm}^{-1}$; ESI-MS: $m / z 464.8\left(\mathrm{M}^{-} 1\right), 466.8\left(\mathrm{M}^{+}+1\right)$, $488.8\left(\mathrm{M}^{+}+\mathrm{Na}\right)$; Anal. Calcd for $\mathrm{C}_{24} \mathrm{H}_{19} \mathrm{ClN}_{2} \mathrm{O}_{4} \mathrm{~S}$ : C 61.73, H 4.10, N 6.00; found C 61.48, H 3.98, N 6.00 .

(E)-N-(5-Chloro-8-hydroxy-2-styrylquinolin-7-yl)-4-chlorobenzenesulfonamide (IIIe): Yield: 52\%; mp: $159-160{ }^{\circ} \mathrm{C} ;{ }^{1} \mathrm{H}-\mathrm{NMR}\left(400 \mathrm{MHz}, \mathrm{DMSO}-d_{6}\right) \delta 7.36(\mathrm{t}, 1 \mathrm{H}, J=7.2 \mathrm{~Hz}, \mathrm{Ar}-H), 7.45(\mathrm{t}, 2 \mathrm{H}, J=7.2$ $\mathrm{Hz}, \operatorname{Ar}-H), 7.45$ (t, $1 \mathrm{H}, J=16 \mathrm{~Hz},-\mathrm{CH}=\mathrm{CH}-), 7.56$ (s, $1 \mathrm{H}, \mathrm{Ar}-H), 7.59$ (d, 2H, $J=7.2 \mathrm{~Hz}, \mathrm{Ar}-H), 7.69$ $(\mathrm{d}, 2 \mathrm{H}, J=7.6 \mathrm{~Hz}, \operatorname{Ar}-H), 7.78(\mathrm{~d}, 2 \mathrm{H}, J=7.2 \mathrm{~Hz}, \operatorname{Ar}-H), 7.82(\mathrm{~d}, 1 \mathrm{H}, J=8.8 \mathrm{~Hz}$, pyridine- $H$ ), 8.28 (d, $1 \mathrm{H}, J=16.4 \mathrm{~Hz},-\mathrm{CH}=\mathrm{CH}-$ ), 8.38 (d, $1 \mathrm{H}, J=8.8 \mathrm{~Hz}$, pyridine- $H$ ), 10.00 (s, br, $1 \mathrm{H}, \mathrm{OH}), 10.26$ (s, br, $1 \mathrm{H}, \mathrm{N} H) ;{ }^{13} \mathrm{C}-\mathrm{NMR}(100 \mathrm{MHz}$, DMSO-d $) \delta 118.7,120.5,122.7,123.4,125.5,127.3,127.8,129.0$, 129.4, 129.7, 133.5, 136.6, 136.7, 138.1, 139.0, 140.0, 146.3, 155.1; IR (KBr): v 3,430, 3,299, 1,633, 1,587, 1,465, 1,328, 1,094 $\mathrm{cm}^{-1}$; ESI-MS: $m / z 468.8\left(\mathrm{M}^{-}-1\right), 470.8\left(\mathrm{M}^{+}+1\right)$.

(E)-N-(5-Chloro-8-hydroxy-2-styrylquinolin-7-yl)-4-methylbenzenesulfonamide (IIIf): Yield: 51\%; mp: $186-188{ }^{\circ} \mathrm{C} ;{ }^{1} \mathrm{H}-\mathrm{NMR}\left(400 \mathrm{MHz}, \mathrm{DMSO}-d_{6}\right) \delta 2.32\left(\mathrm{~s}, 3 \mathrm{H}, \mathrm{CH}_{3}\right), 7.31$ (d, 2H, $\left.J=8.0 \mathrm{~Hz}, \mathrm{Ar}-H\right)$, 7.37 (t, $1 \mathrm{H}, J=7.2 \mathrm{~Hz}, \operatorname{Ar}-H), 7.45$ (t, 2H, $J=7.2 \mathrm{~Hz}, \operatorname{Ar}-H), 7.45$ (d, 1H, $J=15.6 \mathrm{~Hz},-\mathrm{CH}=\mathrm{C} H-$ ), 7.59 (s, 1H, Ar-H), 7.68 (d, 2H, $J=8.2 \mathrm{~Hz}, \operatorname{Ar}-H), 7.69$ (d, 2H, $J=7.2 \mathrm{~Hz}, \operatorname{Ar}-H), 7.79(\mathrm{~d}, 1 \mathrm{H}$, $J=8.8 \mathrm{~Hz}$, pyridine- $H), 8.30(\mathrm{~d}, 1 \mathrm{H}, J=16 \mathrm{~Hz},-\mathrm{C} H=\mathrm{C} H-), 8.38(\mathrm{~d}, 1 \mathrm{H}, J=8.4 \mathrm{~Hz}$, pyridine- $H$ ), 10.03 (s, br, $2 \mathrm{H}, \mathrm{OH}, \mathrm{N} H$ ); ${ }^{13} \mathrm{C}-\mathrm{NMR}\left(100 \mathrm{MHz}, \mathrm{DMSO}-d_{6}\right) \delta 21.4,118.6,121.1,122.6,123.0,124.6$, $127.1,127.3,127.8,129.4,130.0,133.5,136.5,136.7,138.2,138.9,143.5,145.6,155.0$; IR (KBr): $v$ 3,437, 3,295, 1,633, 1,592, 1,462, 1,322, 1,158, $1089 \mathrm{~cm}^{-1}$; ESI-MS: $m / z 448.7\left(\mathrm{M}^{-}-1\right), 450.9\left(\mathrm{M}^{+}+1\right)$. 
(E)-N-(5-Chloro-8-hydroxy-2-styrylquinolin-7-yl)-4-methoxybenzenesulfonamide (IIIg): Yield: 54\%; mp: $193-195{ }^{\circ} \mathrm{C}$; ${ }^{1} \mathrm{H}-\mathrm{NMR}\left(400 \mathrm{MHz}, \mathrm{DMSO}-d_{6}\right) \delta 3.77$ (s, 3H, OCH$), 7.03(\mathrm{~d}, 2 \mathrm{H}, J=8.8 \mathrm{~Hz}, \mathrm{Ar}-$ $H$ ), $7.37(\mathrm{t}, 1 \mathrm{H}, J=7.2 \mathrm{~Hz}, \mathrm{Ar}-H), 7.45$ (t, 2H, $J=8.0 \mathrm{~Hz}, \mathrm{Ar}-H), 7.45$ (d, $1 \mathrm{H}, J=16 \mathrm{~Hz},-\mathrm{CH}=\mathrm{C} H-)$, $7.58(\mathrm{~s}, 1 \mathrm{H}, \operatorname{Ar}-H), 7.69(\mathrm{~d}, 2 \mathrm{H}, J=7.6 \mathrm{~Hz}, \operatorname{Ar}-H), 7.72(\mathrm{~d}, 2 \mathrm{H}, J=8.8 \mathrm{~Hz}, \operatorname{Ar}-H), 7.80(\mathrm{~d}, 1 \mathrm{H}$, $J=8.8 \mathrm{~Hz}$, pyridine- $H$ ), $8.29(\mathrm{~d}, 1 \mathrm{H}, J=16 \mathrm{~Hz},-\mathrm{CH}=\mathrm{C} H-), 8.38(\mathrm{~d}, 1 \mathrm{H}, J=8.4 \mathrm{~Hz}$, pyridine- $H), 9.92$ (s, br, 2H, OH, NH); ${ }^{13} \mathrm{C}-\mathrm{NMR}\left(100 \mathrm{MHz}, \mathrm{DMSO}-d_{6}\right) \delta 56.0,114.7,118.6,121.3,122.5,123.0,124.6$, 127.3, 127.8, 129.3, 129.38, 132.6, 133.5, 136.5, 136.8, 138.9, 145.5, 155.0, 162.8; IR (KBr): v 3,376, 3,236, 1,627, 1,595, 1,499, 1,459, 1,262, 1,155, 1,092 $\mathrm{cm}^{-1}$; ESI-MS: $m / z 464.7\left(\mathrm{M}^{-}-1\right), 466.9\left(\mathrm{M}^{+}+1\right)$, $488.9\left(\mathrm{M}^{+}+\mathrm{Na}\right)$; Anal. Calcd for $\mathrm{C}_{24} \mathrm{H}_{19} \mathrm{ClN}_{2} \mathrm{O}_{4} \mathrm{~S}$ : C 61.73, H 4.10, N 6.00; found C 61.67, H 4.14, N 6.02 .

(E)-N-(5-Chloro-8-hydroxy-2-styrylquinolin-7-yl)benzenesulfonamide (IIIh): Yield: 46\%; mp: 200-201 ${ }^{\circ} \mathrm{C} ;{ }^{1} \mathrm{H}-\mathrm{NMR}\left(400 \mathrm{MHz}, \mathrm{DMSO}-d_{6}\right) \delta 7.37(\mathrm{t}, 1 \mathrm{H}, J=7.2 \mathrm{~Hz}, \mathrm{Ar}-H), 7.45(\mathrm{~d}, 1 \mathrm{H}, J=16 \mathrm{~Hz}$ - $\mathrm{CH}=\mathrm{C} H-), 7.45$ (t, 2H, $J=7.6 \mathrm{~Hz}, \operatorname{Ar}-H), 7.52$ (t, 2H, $J=7.2 \mathrm{~Hz}, \operatorname{Ar}-H), 7.57$ (s, 1H, Ar- $H$ ), 7.60 (t, $1 \mathrm{H}, J=7.2 \mathrm{~Hz}, \operatorname{Ar}-H), 7.70$ (d, $2 \mathrm{H}, J=7.2 \mathrm{~Hz}, \operatorname{Ar}-H), 7.81(\mathrm{~d}, 2 \mathrm{H}, J=7.2 \mathrm{~Hz}, \operatorname{Ar}-H), 7.81(\mathrm{~d}, 1 \mathrm{H}$, $J=8.8 \mathrm{~Hz}$, pyridine- $H$ ), 8.29 (d, $1 \mathrm{H}, J=16 \mathrm{~Hz},-\mathrm{C} H=\mathrm{C} H-), 8.38(\mathrm{~d}, 1 \mathrm{H}, J=8.8 \mathrm{~Hz}$, pyridine- $H), 9.98$ (s, br, $1 \mathrm{H}, \mathrm{OH}), 10.08$ (s, br, $1 \mathrm{H}, \mathrm{N} H) ;{ }^{13} \mathrm{C}-\mathrm{NMR}\left(100 \mathrm{MHz}, \mathrm{DMSO}-d_{6}\right) \delta 118.7,121.0,122.5,123.1$, $124.8,127.0,127.3,127.8,129.4,129.6,133.3,133.5,136.5,136.7,138.9,141.0,145.8,155.1$; IR $(\mathrm{KBr}): v$ 3,304, 1,633, 1,589, 1,506, 1,314, 1,160, 1,090 $\mathrm{cm}^{-1}$; ESI-MS: $m / z 434.7\left(\mathrm{M}^{-}-1\right), 436.9$ $\left(\mathrm{M}^{+}+1\right)$.

(E)-N-(5-Chloro-8-hydroxy-2-styrylquinolin-7-yl)-4-nitrobenzenesulfonamide (IIIi): Yield: 56\%; mp: 247-249 ${ }^{\circ} \mathrm{C}$; ${ }^{1} \mathrm{H}-\mathrm{NMR}\left(400 \mathrm{MHz}, \mathrm{DMSO}-d_{6}\right) \delta 7.36(\mathrm{t}, 1 \mathrm{H}, J=7.2 \mathrm{~Hz}, \mathrm{Ar}-H), 7.45(\mathrm{t}, 2 \mathrm{H}, J=8.0 \mathrm{~Hz}$, $\operatorname{Ar}-H), 7.45(\mathrm{~d}, 1 \mathrm{H}, J=16 \mathrm{~Hz},-\mathrm{C} H=\mathrm{C} H-), 7.58(\mathrm{~s}, 1 \mathrm{H}, \mathrm{Ar}-H), 7.68$ (d, 2H, $J=7.2 \mathrm{~Hz}, \mathrm{Ar}-H), 7.85$ (d, $1 \mathrm{H}, J=8.8 \mathrm{~Hz}$, pyridine- $H$ ), $8.03(\mathrm{dt}, 2 \mathrm{H}, J=8.8 \mathrm{~Hz}, J=2.4 \mathrm{~Hz}, \operatorname{Ar}-H), 8.25$ (d, $1 \mathrm{H}, J=16 \mathrm{~Hz}$, $\mathrm{C} H=\mathrm{C} H-), 8.35$ (dt, $2 \mathrm{H}, J=8.8 \mathrm{~Hz}, J=2.4 \mathrm{~Hz}, \mathrm{Ar}-H), 8.41$ (d, $1 \mathrm{H}, J=8.8 \mathrm{~Hz}$, pyridine- $H$ ), 9.98 (s, br, $1 \mathrm{H}, \mathrm{OH}), 10.53$ (s, br, $1 \mathrm{H}, \mathrm{N} H) ;{ }^{13} \mathrm{C}-\mathrm{NMR}\left(100 \mathrm{MHz}, \mathrm{DMSO}-d_{6}\right) \delta 118.8,120.0,122.8,123.6$, $124.9,125.9,127.3,127.8,128.7,129.4,133.5,136.6,136.7,139.0,146.7,146.8,150.2$, 155.2; IR $(\mathrm{KBr}): v 3,388,3,255,1,519,1,347,1,313,1,172 \mathrm{~cm}^{-1}$; ESI-MS: $m / z 479.6\left(\mathrm{M}^{-}-1\right), 503.9\left(\mathrm{M}^{+}+\mathrm{Na}\right)$; Anal. Calcd for $\mathrm{C}_{23} \mathrm{H}_{16} \mathrm{ClN}_{3} \mathrm{O}_{5} \mathrm{~S}$ : C 57.32, H 3.35, N 8.72; found C 57.56, H 3.46, N 8.66 .

(E)-N-(2-(4-Bromostyryl)-5-chloro-8-hydroxyquinolin-7-yl)-4-methoxybenzenesulfonamide

(IIIj): Yield; 25\%; mp: 206-208 ${ }^{\circ} \mathrm{C}$; ${ }^{1} \mathrm{H}-\mathrm{NMR}\left(400 \mathrm{MHz}, \mathrm{DMSO}-d_{6}\right) \delta 3.76$ (s, 3H, OCH ), 7.02 (dt, 2H, $J=8.8 \mathrm{~Hz}, J=2.4 \mathrm{~Hz}, \mathrm{Ar}-H), 7.47(\mathrm{~d}, 1 \mathrm{H}, J=16 \mathrm{~Hz},-\mathrm{CH}=\mathrm{CH}-), 7.60$ (s, 1H, Ar- $H), 7.62$ (s, 4H, Ar$H), 7.72(\mathrm{dt}, 2 \mathrm{H}, J=8.8 \mathrm{~Hz}, J=2.4 \mathrm{~Hz}, \operatorname{Ar}-H), 7.76(\mathrm{~d}, 1 \mathrm{H}, J=8.8 \mathrm{~Hz}$, pyridine- $H$ ), $8.26(\mathrm{~d}, 1 \mathrm{H}$, $J=16 \mathrm{~Hz},-\mathrm{C} H=\mathrm{C} H-), 8.36(\mathrm{~d}, 1 \mathrm{H}, J=8.8 \mathrm{~Hz}$, pyridine- $H), 9.94(\mathrm{~s}, \mathrm{br}, 2 \mathrm{H}, \mathrm{O} H, \mathrm{~N} H) ;{ }^{13} \mathrm{C}-\mathrm{NMR}(100$ MHz, DMSO- $\left.d_{6}\right) \delta 56.0,114.7,118.7,121.3,122.4,122.6,123.0,124.7,128.1,129.3,129.7,132.3$, 132.6, 122.6, 135.1, 136.0, 138.9, 145.5, 154.7, 162.8; IR (KBr): v 3,291, 1,594, 1,463, 1,261, 1,157, 1,090 $\mathrm{cm}^{-1}$; ESI-MS: m/z $544.7\left(\mathrm{M}^{-}-1\right)$; Anal. Calcd for $\mathrm{C}_{24} \mathrm{H}_{18} \mathrm{BrClN}_{2} \mathrm{O}_{4} \mathrm{~S}$ : C 52.81, H 3.32, N 5.13; found C 52.73, H 3.45, N 5.11. 
(E)-N-(2-(4-Bromostyryl)-5-chloro-8-hydroxyquinolin-7-yl)-4-methylbenzenesulfonamide

(IIIk):

Yield: 51\%; mp: $234-236{ }^{\circ} \mathrm{C}$; ${ }^{1} \mathrm{H}-\mathrm{NMR}\left(400 \mathrm{MHz}, \mathrm{DMSO}-d_{6}\right) \delta 2.32\left(\mathrm{~s}, 3 \mathrm{H}, \mathrm{CH}_{3}\right), 7.31(\mathrm{~d}, 2 \mathrm{H}$, $J=8.0 \mathrm{~Hz}, \mathrm{Ar}-H), 7.48(\mathrm{~d}, 1 \mathrm{H}, J=16 \mathrm{~Hz},-\mathrm{CH}=\mathrm{C} H-), 7.59$ (s, $1 \mathrm{H}, \mathrm{Ar}-H), 7.63-7.64$ (m, 4H, Ar- $H$ ), $7.68(\mathrm{~d}, 2 \mathrm{H}, J=8.0 \mathrm{~Hz}, \operatorname{Ar}-H), 7.78(\mathrm{~d}, 1 \mathrm{H}, J=8.8 \mathrm{~Hz}$, pyridine- $H), 8.29(\mathrm{~d}, 1 \mathrm{H}, J=16 \mathrm{~Hz}$, $\mathrm{C} H=\mathrm{C} H-), 8.38(\mathrm{~d}, 1 \mathrm{H}, J=8.4 \mathrm{~Hz}$, pyridine- $H), 9.98(\mathrm{~s}, \mathrm{br}, 1 \mathrm{H}, \mathrm{O} H), 10.04(\mathrm{~s}, \mathrm{br}, 1 \mathrm{H}, \mathrm{N} H) ;{ }^{13} \mathrm{C}-\mathrm{NMR}$ $\left(100 \mathrm{MHz}, \mathrm{DMSO}-d_{6}\right) \delta 21.4,118.6,121.2,122.4,122.7,123.1,124.8,127.1,128.1,129.7,130.0$, 132.4, 133.6, 135.1, 136.1, 138.2, 138.9, 143.5, 145.5, 154.7; IR (KBr): v 3,291, 1,634, 1,585, 1,460, $1,310,1,158 \mathrm{~cm}^{-1}$; ESI-MS: $m / z 528.6\left(\mathrm{M}^{-}-1\right)$.

(E)-N-(2-(4-Bromostyryl)-5-chloro-8-hydroxyquinolin-7-yl)benzenesulfonamide (IIIl): Yield: 38\%; mp: $238-239{ }^{\circ} \mathrm{C} ;{ }^{1} \mathrm{H}-\mathrm{NMR}\left(400 \mathrm{MHz}, \mathrm{DMSO}-d_{6}\right) \delta 7.48(\mathrm{~d}, 1 \mathrm{H}, J=16 \mathrm{~Hz},-\mathrm{CH}=\mathrm{CH}-), 7.52$ (t, 2H, $J=7.2 \mathrm{~Hz}, \operatorname{Ar}-H), 7.58(\mathrm{~s}, 1 \mathrm{H}, \operatorname{Ar}-H), 7.60$ (t, 1H, $J=8.4 \mathrm{~Hz}, \operatorname{Ar}-H), 7.64$ (s, 4H, Ar-H), 7.65 (d, 2H, $J=9.6 \mathrm{~Hz}, \operatorname{Ar}-H), 7.78(\mathrm{~d}, 1 \mathrm{H}, J=8.8 \mathrm{~Hz}$, pyridine- $H), 7.80(\mathrm{~d}, 2 \mathrm{H}, J=7.2 \mathrm{~Hz}, \operatorname{Ar}-H), 8.28(\mathrm{~d}, 1 \mathrm{H}$, $J=16 \mathrm{~Hz},-\mathrm{CH}=\mathrm{C} H-), 8.39$ (d, $1 \mathrm{H}, J=8.8 \mathrm{~Hz}$, pyridine- $H$ ), 9.98 (s, br, $1 \mathrm{H}, \mathrm{OH}), 10.14$ (s, br, $1 \mathrm{H}$, $\mathrm{N} H) ;{ }^{13} \mathrm{C}-\mathrm{NMR}\left(100 \mathrm{MHz}, \mathrm{DMSO}-d_{6}\right) \delta 118.6,121.0,122.4,122.7,123.2,124.9,127.0,128.1,129.5$, 129.7, 132.4, 133.3, 133.6, 135.2, 136.0, 138.9, 141.0, 145.8, 154.7; IR (KBr): v 2,924, 2,853, 1,459, 1,310, 1,159 $\mathrm{cm}^{-1}$; ESI-MS: $\mathrm{m} / z$ $514.5\left(\mathrm{M}^{-}-1\right), 538.9\left(\mathrm{M}^{+}+\mathrm{Na}\right)$.

(E)-N-(2-(4-Bromostyryl)-5-chloro-8-hydroxyquinolin-7-yl)-4-chlorobenzenesulfonamide

(IIIm): Yield: 35\%; mp: $238-240{ }^{\circ} \mathrm{C}$; ${ }^{1} \mathrm{H}-\mathrm{NMR}\left(400 \mathrm{MHz}, \mathrm{DMSO}-d_{6}\right) \delta 7.44$ (d, $1 \mathrm{H}, J=16 \mathrm{~Hz},-\mathrm{CH}=\mathrm{C} H-$ ), $7.57(\mathrm{~s}, 1 \mathrm{H}, \operatorname{Ar}-H), 7.60(\mathrm{~d}, 2 \mathrm{H}, J=7.2 \mathrm{~Hz}, \operatorname{Ar}-H), 7.61(\mathrm{~s}, 4 \mathrm{H}, \operatorname{Ar}-H), 7.76(\mathrm{~d}, 1 \mathrm{H}, J=8.8 \mathrm{~Hz}$, pyridine- $H$ ), $7.79(\mathrm{~d}, 2 \mathrm{H}, J=7.2 \mathrm{~Hz}, \mathrm{Ar}-H), 8.24(\mathrm{~d}, 1 \mathrm{H}, J=16 \mathrm{~Hz},-\mathrm{CH}=\mathrm{CH}-), 8.35(\mathrm{~d}, 1 \mathrm{H}, J=8.8$ $\mathrm{Hz}$, pyridine- $H$ ), 10.15 (s, br, 2H, OH, NH); ${ }^{13} \mathrm{C}-\mathrm{NMR}\left(100 \mathrm{MHz}, \mathrm{DMSO}-d_{6}\right) \delta 118.7,120.5,122.4$, 122.7, 123.4, 125.6, 128.1, 129.0, 129.7, 132.3, 133.6, 135.2, 136.0, 138.1, 138.9, 139.9, 146.3, 154.8; IR (KBr): $v 3,435,3,292,1,633,1,461,1,326,1,161 \mathrm{~cm}^{-1}$; ESI-MS: $m / z 548.5\left(\mathrm{M}^{-}-1\right), 572.9\left(\mathrm{M}^{+}+\mathrm{Na}\right)$.

(E)-N-(2-(4-Hydroxystyryl)-5-chloro-8-hydroxyquinolin-7-yl)-4-methoxybenzenesulfonamide (IIIn): Yield: 33\%; mp: $212-213{ }^{\circ} \mathrm{C}$; ${ }^{1} \mathrm{H}-\mathrm{NMR}\left(400 \mathrm{MHz}, \mathrm{DMSO}-d_{6}\right) \delta 3.76\left(\mathrm{~s}, 3 \mathrm{H}, \mathrm{OCH}_{3}\right), 6.84(\mathrm{~d}, 2 \mathrm{H}$, $J=8.8 \mathrm{~Hz}, \operatorname{Ar}-H), 7.01(\mathrm{dt}, 2 \mathrm{H}, J=7.2 \mathrm{~Hz}, J=2.4 \mathrm{~Hz}, \mathrm{Ar}-H), 7.21(\mathrm{~d}, 1 \mathrm{H}, J=16 \mathrm{~Hz},-\mathrm{C} H=\mathrm{C} H-)$, $7.53(\mathrm{~d}, 2 \mathrm{H}, J=8.4 \mathrm{~Hz}, \operatorname{Ar}-H), 7.55(\mathrm{~s}, 1 \mathrm{H}, \mathrm{Ar}-H), 7.72(\mathrm{dt}, 2 \mathrm{H}, J=7.2 \mathrm{~Hz}, J=2.4 \mathrm{~Hz}, \operatorname{Ar}-H), 7.73(\mathrm{~d}$, $1 \mathrm{H}, J=8.8 \mathrm{~Hz}$, pyridine- $H$ ), $8.17(\mathrm{~d}, 1 \mathrm{H}, J=16.4 \mathrm{~Hz},-\mathrm{CH}=\mathrm{CH}-), 8.31(\mathrm{~d}, 1 \mathrm{H}, J=8.8 \mathrm{~Hz}$, pyridine$H$ ), 9.80-9.95 (m, br, 3H, OH, NH); ${ }^{13} \mathrm{C}-\mathrm{NMR}$ (100 MHz, DMSO-d $) \delta 56.0,114.7,116.3,118.6$, 121.2, 122.2, 122.7, 124.0, 124.2, 127.8, 129.3, 129.5, 132.6, 133.2, 136.7, 138.9, 145.3, 155.6, 159.0, 162.8; IR (KBr): $v$ 3,430, 3,251, 1,630, 1,590, 1,498, 1,438, 1,319, 1,264, 1,147 cm $\mathrm{cm}^{-1}$ ESI-MS: $\mathrm{m} / \mathrm{z}$ $480.7\left(\mathrm{M}^{-}-1\right), 483.0\left(\mathrm{M}^{+}+1\right), 505.0\left(\mathrm{M}^{+}+\mathrm{Na}\right)$; Anal. Calcd for $\mathrm{C}_{24} \mathrm{H}_{19} \mathrm{ClN}_{2} \mathrm{O}_{5} \mathrm{~S}: \mathrm{C}$ 59.69, H 3.97, N 5.80; found C 59.41, H 4.05, N 5.64.

(E)-N-(2-(4-Hydroxystyryl)-5-chloro-8-hydroxyquinolin-7-yl)-4-methylbenzenesulfonamide

(IIIo): Yield: 31\%; mp: $214-215{ }^{\circ} \mathrm{C}$; ${ }^{1} \mathrm{H}-\mathrm{NMR}\left(400 \mathrm{MHz}, \mathrm{DMSO}-d_{6}\right) \delta 2.32\left(\mathrm{~s}, 3 \mathrm{H}, \mathrm{CH}_{3}\right), 6.84(\mathrm{~d}, 2 \mathrm{H}$, $J=8.4 \mathrm{~Hz}, \mathrm{Ar}-H), 7.21(\mathrm{~d}, 1 \mathrm{H}, J=16 \mathrm{~Hz},-\mathrm{C} H=\mathrm{CH}-), 7.31(\mathrm{~d}, 2 \mathrm{H}, J=8.0 \mathrm{~Hz}, \mathrm{Ar}-H), 7.53(\mathrm{~d}, 2 \mathrm{H}$, $J=8.8 \mathrm{~Hz}, \operatorname{Ar}-H), 7.55$ (s, $1 \mathrm{H}, \operatorname{Ar}-H), 7.68(\mathrm{~d}, 2 \mathrm{H}, J=8.0 \mathrm{~Hz}, \operatorname{Ar}-H), 7.73(\mathrm{~d}, 1 \mathrm{H}, J=8.8 \mathrm{~Hz}$, pyridine- $H$ ), 8.19 (d, $1 \mathrm{H}, J=16 \mathrm{~Hz},-\mathrm{CH}=\mathrm{CH}-), 8.32(\mathrm{~d}, 1 \mathrm{H}, J=8.4 \mathrm{~Hz}$, pyridine- $H), 9.83(\mathrm{~s}, 1 \mathrm{H}$, 
$\mathrm{OH}), 9.89(\mathrm{~s}, 1 \mathrm{H}, \mathrm{OH}), 10.02(\mathrm{~s}, 1 \mathrm{H}, \mathrm{N} H) ;{ }^{13} \mathrm{C}-\mathrm{NMR}\left(100 \mathrm{MHz}, \mathrm{DMSO}-d_{6}\right) \delta 21.4,116.3,118.6$, 121.0, 122.3, 122.7, 123.9, 124.2, 127.1, 127.8, 129.5, 130.0, 133.3, 136.8, 138.2, 138.9, 143.5, 145.4, 155.6, 159.0; IR (KBr): v 3,434, 3,258, 1,589, 1,515, 1,458, 1,279, 1,185, 1,150 cm ${ }^{-1}$; ESI-MS: $\mathrm{m} / z$ $464.8\left(\mathrm{M}^{-}-1\right), 466.9\left(\mathrm{M}^{+}+1\right), 488.9\left(\mathrm{M}^{+}+\mathrm{Na}\right)$; Anal. Calcd for $\mathrm{C}_{24} \mathrm{H}_{19} \mathrm{ClN}_{2} \mathrm{O}_{4} \mathrm{~S}: \mathrm{C}$ 61.73, $\mathrm{H}$ 4.10, N 6.00 .

(E)-N-[2-(4-Hydroxystyryl)-5-chloro-8-hydroxyquinolin-7-yl]benzenesulfonamide (IIIp): Yield: 25\%; mp: $191-193{ }^{\circ} \mathrm{C} ;{ }^{1} \mathrm{H}-\mathrm{NMR}\left(400 \mathrm{MHz}, \mathrm{DMSO}_{6}\right) \delta 6.84(\mathrm{~d}, 2 \mathrm{H}, J=8.8 \mathrm{~Hz}, \operatorname{Ar}-H), 7.22(\mathrm{~d}, 1 \mathrm{H}$, $J=16 \mathrm{~Hz},-\mathrm{C} H=\mathrm{C} H-), 7.50-7.54(\mathrm{~m}, 5 \mathrm{H}, \mathrm{Ar}-H), 7.60(\mathrm{tt}, 1 \mathrm{H}, J=6.8 \mathrm{~Hz}, J=2.0 \mathrm{~Hz}, \mathrm{Ar}-H), 7.74(\mathrm{~d}$, $1 \mathrm{H}, J=8.8 \mathrm{~Hz}$, pyridine- $H$ ), $7.79(\mathrm{dt}, 2 \mathrm{H}, J=7.2 \mathrm{~Hz}, J=2.0 \mathrm{~Hz}, \operatorname{Ar}-H), 8.19$ (d, $1 \mathrm{H}, J=16 \mathrm{~Hz}$, $\mathrm{C} H=\mathrm{C} H-), 8.33(\mathrm{~d}, 1 \mathrm{H}, J=8.8 \mathrm{~Hz}$, pyridine- $H), 9.86(\mathrm{~s}, 1 \mathrm{H}, \mathrm{OH}), 9.91(\mathrm{~s}, 1 \mathrm{H}, \mathrm{OH}), 10.15(\mathrm{~s}, 1 \mathrm{H}$, $\mathrm{N} H) ;{ }^{13} \mathrm{C}$ - NMR (100 MHz, DMSO- $\left.d_{6}\right) \delta 116.3,118.6,120.8,122.3,122.8,1238,124.4,127.0,127.8$, 129.5, 129.6, 133.3, 136.8, 138.8, 140.9, 145.6, 155.6, 159.0; IR (KBr): v 3,392, 1,625,1,585, 1,513, 1,455, 1,337, 1,154, 1,091 $\mathrm{cm}^{-1}$; ESI-MS: $m / z 450.7\left(\mathrm{M}^{-}-1\right), 475.0\left(\mathrm{M}^{+}+\mathrm{Na}\right)$.

(E)-N-(2-(4-Hydroxystyryl)-5-chloro-8-hydroxyquinolin-7-yl)-4-chlorobenzenesulfonamide

(IIIq):

Yield: $16 \%$; mp: $189-191{ }^{\circ} \mathrm{C}$; ${ }^{1} \mathrm{H}-\mathrm{NMR}\left(400 \mathrm{MHz}\right.$, acetone- $\left.d_{6}\right) \delta 6.91(\mathrm{~d}, 2 \mathrm{H}, J=8.4 \mathrm{~Hz}, \mathrm{Ar}-H), 7.24$ $(\mathrm{d}, 1 \mathrm{H}, J=16 \mathrm{~Hz},-\mathrm{CH}=\mathrm{CH}-), 7.53$ (d, 2H, $J=8.4 \mathrm{~Hz}, \mathrm{Ar}-H), 7.57$ (d, 2H, $J=8.8 \mathrm{~Hz}, \mathrm{Ar}-H), 7.77$ (s, $1 \mathrm{H}, \operatorname{Ar}-H), 7.79(\mathrm{~d}, 1 \mathrm{H}, J=8.8 \mathrm{~Hz}$, pyridine- $H), 7.86(\mathrm{~d}, 2 \mathrm{H}, J=8.4 \mathrm{~Hz}, \operatorname{Ar}-H), 8.05(\mathrm{~d}, 1 \mathrm{H}, J=16$ $\mathrm{Hz},-\mathrm{C} H=\mathrm{C} H-), 8.40(\mathrm{~d}, 1 \mathrm{H}, J=8.8 \mathrm{~Hz}$, pyridine- $H), 8.82(\mathrm{~s}, \mathrm{br}, 3 \mathrm{H}, \mathrm{OH}, \mathrm{O} H, \mathrm{~N} H) ;{ }^{13} \mathrm{C}-\mathrm{NMR}(100$ $\mathrm{MHz}$, acetone- $\left.d_{6}\right) \delta 115.8,119.6,120.2,121.6,122.8,123.4,123.8,128.1,128.9,129.1,129.2,133.1$, 136.73, 136.2, 138.4, 138.5, 144.0, 155.8, 158.6; IR (KBr): v 3,252, 1,630, 1,514, 1,274, 1,168, 1,091 $\mathrm{cm}^{-1}$; ESI-MS: $m / z 484.8\left(\mathrm{M}^{-}-1\right), 486.9\left(\mathrm{M}^{+}+1\right), 508.9\left(\mathrm{M}^{+}+\mathrm{Na}\right)$.

HIV-IN inhibitory activity evaluation

Compounds III $\left(5 \times 10^{-5} \mathrm{mmol}\right)$ diluted in DMSO $(1 \mathrm{~mL})$ were pre-incubated with $800 \mathrm{ng}$ IN at $37{ }^{\circ} \mathrm{C}$ in the reaction buffer in the absence of $\mathrm{Mn}^{2+}$ for $10 \mathrm{~min}$. Subsequently, 1.5 pmol donor DNA and 9 pmol target DNA were added and the reaction was initiated by the addition of $\mathrm{Mn}^{2+}(10 \mathrm{mmol} / \mathrm{L})$ into the final reaction volume. The reactions were carried out at $37{ }^{\circ} \mathrm{C}$ for $1 \mathrm{~h}$ and subsequent detection procedure was applied to detect the assay signals. In these experiments, baicalein, a known IN inhibitor with both viral replication inhibitory effect in vivo and IN reaction activities inhibitory effect in vitro, was used as the control compound (positive control), whereas 10\% DMSO solution without sample was set as the drug-free control (negative control).

\section{Conclusions}

In summary, on the basis of our previous CoMFA analysis of styrylquinoline derivatives, we have designed and synthesized for the first time a series of $\mathrm{N}$-[(2-substituted-styryl)-5-chloro-8hydroxyquinolin-7-yl]-benzenesulfonamide derivatives. The structures of these compounds were characterized and their HIV IN inhibitory activities were evaluated. Results indicate that most of the title compounds exhibit moderate inhibitory activity. Improved inhibitory activity can be achieved when free hydroxyl at the styryl moiety and nitro group at the benzenesulfonamide moiety are present. 


\section{Acknowledgements}

This work was supported by Grants from the National Natural Science Foundation of China (No. 20402001, 20772010), the National Basic Research Program of China (No. 2009CB930200) and Beijing Novel Project (No. 2005B10).

\section{References and Notes}

1. Debyser, Z.; Cherepanov, P.; Maele, B.V.; De Clercq, E. Witvrouw, M. In search of authentic inhibitors of HIV-1 integration. Antivir. Chem. Chemother. 2002, 13, 1-15.

2. De Clercq, E. Strategies in the design of antiviral drugs. Nat. Rev. Drug Disc. 2002, 1, 13-25.

3. Reader, J.C. Automation in medicinal chemistry. Curr. Top. Med. Chem. 2004, 4, 671-686

4. Neamati, N. Patented small molecule inhibitors of HIV-1 integrase: A 10-year saga. Exp. Opin. Ther. Pat. 2002, 12, 709-724.

5. Mekouar, K.; Mouscadet, J.F.; Desmaele, D.; Subra, F.; Leh, H.; Savoure, D.; Auclair, C.; d'Angelo, J. Styrylquinoline derivatives: A new class of potent HIV-1 integrase inhibitors that block HIV-1 replication in CEM cells. J. Med. Chem. 1998, 41, 2846-2857.

6. Zouhiri, F.; Mouscadet, J.F.; Mekouar, K.; Desmaele, D.; Savoure, D.; Leh, H.; Subra, F.; Bret, M.L.; Auclair, C.; d'Angelo, J. Structure-activity relationships and binding mode of styrylquinolines as potent inhibitors of HIV-1 integrase and replication of HIV-1 in cell culture. $J$. Med. Chem. 2000, 43, 1533-1540.

7. Polanski, J.; Zouhiri, F.; Jeanson, L.; Desmaele, D.; d'Angelo, J.; Mouscadet, J.-F.; Gieleciak, R.; Gasteiger, J.; Bret, L.M. Use of the Kohonen neural network for rapid screening of ex vivo antiHIV activity of styrylquinolines. J. Med. Chem. 2002, 45, 4647-4654.

8. Normand-Baylea, M.; Bénarda, C.; Zouhiria, F.; Mouscadetc, J.F.; Lehb, H.; Thomasb, C.M.; Mbembac, G.; Desmaëlea, D.; d'Angeloa, J. New HIV-1 replication inhibitors of the styrylquinoline class bearing aroyl/acyl groups at the C-7 position: Synthesis and biological activity. Bioorg. Med. Chem. Lett. 2005, 15, 4019-4022.

9. Ma, X.H.; Zhang, X.Y.; Tan, J.J. Chen, W.Z.; Wang, C.X. Exploring binding mode for styrylquinoline HIV-1 integrase inhibitors using comparative molecular field analysis and docking studies. Acta Pharmacol. Sin. 2004, 25, 950-958.

10. Zeng, C.C.; Niu, L.T.; Ping, D.W.; Zhong, R.G. Design and synthesis of 2-styrylquinoline-7sulfonamide derivatives as potential HIV integrase inhibitors (in Chinese). Chin. J. Org. Chem. 2009, 29, 1105-1114.

11. Yoo, H.; Lee, J.Y.; Park, J.H.; Chung, B.Y.; Lee, Y.S. Synthesis of styrylbenzofuran derivatives as styrylquinoline analogues for HIV-1 integrase inhibitors. Farmaco 2003, 58, 1243-1250.

12. Kurita, K. Selectivity in tosylation of $o$-aminophenol by choice of tertiary amine Chem. Ind. 1974, 345-346.

13. Sellarajah, S.; Lekishvili, T.; Bowring, C.; Thompsett, A.R.; Rudyk, H.; Birkett, C.R.; Brown, D.R.; Gilbert, I.H. Synthesis of analogues of congo red and evaluation of their anti-prion activity. J. Med. Chem. 2004, 47, 5515-5534. 
14. He, H.Q.; Ma, X.H.; Liu, B.; Zhang, X.Y.; Chen, W.Z.; Wang, C.X.; Cheng, S.H. A novel highthroughput format assay for HIV-1 integrase strand transfer reaction using magnetic beads. Acta Pharmacol. Sin. 2008, 29, 397-404.

15. Zouhiri, F.; Danet, M.; Bénard, C.; Normand-Bayle, M.; Mouscadet, J.F.; Leh, H.; Thomas, C.M.; Mbemba, G.; d'Angelo, J.; Desmaële, D. Tetrahedron Lett. 2005, 46, 2201-2205.

16. Weizmann, M.; Bograchov, E. Derivatives of 5-chloro-8-hydroxyquinoline. J. Am. Chem. Soc. 1947, 69, 1222-1223.

Sample Availability: Samples of the compounds IIIa-m are available from the authors.

(C) 2010 by the authors; licensee Molecular Diversity Preservation International, Basel, Switzerland. This article is an open-access article distributed under the terms and conditions of the Creative Commons Attribution license (http://creativecommons.org/licenses/by/3.0/). 